\title{
The Future Role of Inorganic Crystal Scintillators in Dark Matter Investigations
}

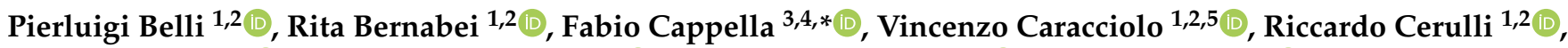

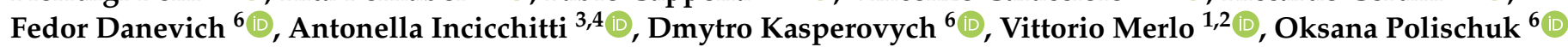 \\ and Vladimir Tretyak ${ }^{6}$ (D) \\ 1 INFN, Sezione di Roma Tor Vergata, 00133 Rome, Italy; pierluigi.belli@roma2.infn.it (P.B.); \\ rita.bernabei@roma2.infn.it (R.B.); vincenzo.caracciolo@roma2.infn.it (V.C.); \\ riccardo.cerulli@roma2.infn.it (R.C.); vittorio.merlo@roma2.infn.it (V.M.) \\ 2 Dipartimento di Fisica, Università di Roma Tor Vergata, 00133 Rome, Italy \\ 3 INFN, Sezione di Roma, 00185 Rome, Italy; antonella.incicchitti@roma1.infn.it \\ 4 Dipartimento di Fisica, Università di Roma La Sapienza, 00185 Rome, Italy \\ 5 INFN, Laboratori Nazionali del Gran Sasso, 67100 Assergi, Italy \\ 6 Institute for Nuclear Research of NASU, 03028 Kyiv, Ukraine; danevich@kinr.kiev.ua (F.D.); \\ casper.phys@gmail.com (D.K.); polischuk@kinr.kiev.ua (O.P.); tretyak@kinr.kiev.ua (V.T.) \\ * Correspondence: fabio.cappella@roma1.infn.it
}

\section{check for}

updates

Citation: Belli, P.; Bernabei, R.;

Cappella, F.; Caracciolo, V.; Cerulli, R.;

Danevich, F.A.; Incicchitti, A.;

Kasperovych, D.V.; Merlo, V.;

Polischuk, O.G.; Tretyak, V.I. The

Future Role of Inorganic Crystal

Scintillators in Dark Matter

Investigations. Instruments 2021, 5, 16.

https:/ /dx.doi.org/10.3390/

instruments5020016

Academic Editor: Andrea Messina

Received: 5 February 2021

Accepted: 21 April 2021

Published: 28 April 2021

Publisher's Note: MDPI stays neutral with regard to jurisdictional claims in published maps and institutional affiliations.

Copyright: (C) 2021 by the authors. Licensee MDPI, Basel, Switzerland. This article is an open access article distributed under the terms and conditions of the Creative Commons Attribution (CC BY) license (https:/ / creativecommons.org/licenses/by/ $4.0 /)$.
Abstract: Crystal scintillators and in particular inorganic scintillators play an important role in the investigation of Dark Matter (DM) and other rare processes. The investigation of a DM signature, as the annual modulation, or the directionality technique requires the use of highly radiopure detectors able to explore the very low energy region maintaining a high stability of the running conditions. In this paper, the cases of $\mathrm{NaI}(\mathrm{Tl}), \mathrm{ZnWO}_{4}$ and $\mathrm{SrI}_{2}(\mathrm{Eu})$ crystal scintillators are described in the framework of our activities at the Gran Sasso National Laboratory of the INFN. Their role, the obtained results in DM investigation, as well as their potential and perspectives for the future are reviewed.

Keywords: dark matter detector; directional detection; underground experiments

\section{Introduction}

Astrophysical observations have revealed the presence of DM on all astrophysical scales and much experimental and theoretical evidence supports arguments that a large fraction of DM should be in the form of relic DM particles. Crystal scintillators and in particular inorganic scintillators play an important role in the investigation of DM and other rare processes. In particular, the investigation of a DM signature requires the use of highly radiopure detectors able to explore the very low energy region with a high stability of the running conditions. The paper will review the cases of the $\mathrm{NaI}(\mathrm{Tl}), \mathrm{ZnWO}_{4}$ and $\mathrm{SrI}_{2}(\mathrm{Eu})$ crystal scintillators, developed and used in the framework of our activities at the Gran Sasso National Laboratory of the INFN.

The best qualities offered by inorganic scintillators are due to their physical characteristics (high $\mathrm{Z}$ materials i.e., high detection efficiency for gamma quanta, physical form, chemical and mechanical stability) and to their luminescent characteristics (emission wavelength, high light yield, good linearity, suitable decay time, and good radiation hardness). In particular, the high radio-purity, high light yield, the possible choice of different target nuclei, the stability of performance and the reproducibility of the results make these detector particularly suitable for the study of DM annual modulation (DM-AM) signature [1,2].

Ultra-low-background (ULB) $\mathrm{NaI}(\mathrm{Tl})$ crystal scintillators have been used by the DAMA/NaI, DAMA/LIBRA-phase1 and DAMA/LIBRA-phase2 [3-7] experiments at the Gran Sasso National Laboratory of INFN (LNGS) for the direct search for DM. These 
experiments have obtained evidence at a $12.9 \sigma$ confidence level of a signal satisfying all the requirements of the model independent DM-AM signature. The selected approach and target assure sensitivity both to DM candidate particles inducing nuclear recoils, as well as to those for which the products of the interaction have electromagnetic nature (mainly electrons). Moreover, the observed signal is compatible with a wide set of scenarios regarding the nature of the DM candidates and related astrophysical, nuclear, and particle physics (see, e.g., Ref. [8] and references therein).

Other features of inorganic scintillators, such as channeling and anisotropy, make them very promising for the investigation of the directionality of DM [9], potentially effective for those DM candidate particles able to induce nuclear recoils. The directionality approach is based on the study of the correlation between the DM particles' arrival direction, through the induced nuclear recoils, and the Earth motion in the galactic rest frame. A detector sensitive to nuclear recoils direction is then required and a promising option is represented by the anisotropic scintillators, where the scintillation properties (quenching and scintillation decay kinetics) depend on the direction of the detected heavy particles relatively to the crystal axes. Among the anisotropic crystal scintillators, the zinc tungstate $\left(\mathrm{ZnWO}_{4}\right)$ offers very interesting features and its potential for DM investigation with the directionality technique is explored in the framework of the ADAMO project (Anisotropic detectors for DArk Matter Observation) [10-12].

Finally, other scintillators became attractive in the last few years, such as $\operatorname{SrI}_{2}(\mathrm{Eu})$ crystal scintillators because of their high light output ( 100,000 photons $/ \mathrm{MeV})$ and of the absence of natural long-living radioactive isotopes. These and other features make $\mathrm{SrI}_{2}(\mathrm{Eu})$ scintillators promising for low counting experiments such as the search for double $\beta$ decay $\left({ }^{84} \mathrm{Sr}\right.$ is a candidate) and the investigation of $\mathrm{DM}$, which requires a very low energy threshold. In particular, in ${ }^{84} \mathrm{Sr}$, the double electron capture $(2 \varepsilon)$ and electron capture with emission of positron $\left(\varepsilon \beta^{+}\right)$are energetically allowed. These decays have never been observed and the half-life limits are of the order of $10^{16} \mathrm{y}$ [13]. Studies of $2 \varepsilon$ and $\varepsilon \beta^{+}$decays are important because the observation of neutrinoless mode of such a process could help to distinguish between the mechanisms of neutrinoless $2 \beta^{-}$decay: whether it is due to the light Majorana neutrino mass or due to the right-handed currents' admixture in the weak interaction [14].

In the following section, the strategies to develop ULB scintillators will be discussed. Then, the cases of $\mathrm{NaI}(\mathrm{Tl}), \mathrm{ZnWO}_{4}$ and $\mathrm{SrI}_{2}(\mathrm{Eu})$ crystal scintillators and their application in the investigation of DM will be reviewed, focusing the attention on the results obtained by DAMA/LIBRA using $\mathrm{NaI}(\mathrm{Tl})$ detectors and on the recent measurements of the ADAMO project, utilizing $\mathrm{ZnWO}_{4}$ anisotropic detectors.

\section{Radiopurity of Crystal Scintillators}

A wide set of prerequisites has to be fulfilled to obtain the best sensitivity in the investigation of DM, e.g.,: (i) the choice of a suitable underground laboratory for cosmic background reduction; (ii) a suitable shield to be used underground to prevent background from environmental radioactivity (gammas, muons, neutrons, radon, etc.); (iii) the choice of a suitable set-up configuration, e.g., with multiple detectors that offers the possibility to discriminate background events; (iv) selection of low radioactivity materials;, etc.. Nevertheless, the most effective way to obtain the highest sensitivities is closely related to the achievement of an extreme radio-purity in the used crystal scintillators. This process can be slow and challenging.

In inorganic scintillators, the presence of natural radionuclides from ${ }^{232} \mathrm{Th},{ }^{238} \mathrm{U}$ and ${ }^{235} \mathrm{U}$ families and ${ }^{40} \mathrm{~K}$, and other cosmogenic and/or anthropogenic radionuclides is common. Some materials contain elements with radioactive isotopes, like ${ }^{113} \mathrm{Cd}$ in $\mathrm{CdWO}_{4}$, ${ }^{138} \mathrm{La}$ in $\mathrm{LaCl}_{3}$ and $\mathrm{LaBr}_{3}$, and so on [15].

Achieving the high radiopurity goal relies on a highly specialized experience and a long, delicate work. In fact, in addition to the prerequisites already mentioned, it is important to have experience on natural, cosmogenic and anthropogenic contaminants, which can 
be different even in batches of the same material. For each used material, it is important to know origin, history, and manipulation processes. Furthermore, particular attention has to be dedicated to the procedures and protocols for the project/storage/cleaning/installation of the experiment, and to the specific experimental optimizations, which can be pursued for the radio-purity goal. Finally, a deep experience on the measurements and analysis techniques, especially developed for this field, is mandatory. Similar developments and measurements follow different subsequent steps, which can be summarized for inorganic scintillators in:

- $\quad$ Pre-selection and analyses of materials by suitable techniques.

- $\quad$ Selection of growing processes.

- $\quad$ Selection of additives.

- Investigation on radioactive contaminants.

- Chemical/physical purification of selected materials.

- Growing protocols.

- Identification of the optimal procedures and tools for crystal processing.

- Selection of housing and materials in the surrounding environment.

- Protocols and procedures for the project/storage/cleaning/installation of the experiment, from the crystal growth up to the installation deep underground.

- Underground tests.

In case of a positive outcome producing an improvement in radio-purity, the production of detectors can start. Otherwise, the critical problems have to be solved with targeted solutions.

Regarding the measurement techniques of radioactive contaminations, it is worth mentioning that the ULB HP-Ge detectors can reach sensitivity at the level of $\mathrm{mBq} / \mathrm{kg}$ (generally ppb) for ${ }^{228} \mathrm{Th}\left({ }^{232} \mathrm{Th}\right),{ }^{226} \mathrm{Ra}\left({ }^{238} \mathrm{U}\right),{ }^{227} \mathrm{Ac}\left({ }^{235} \mathrm{U}\right)$ and ${ }^{40} \mathrm{~K}$. This method is commonly used to measure gamma emitters in samples of materials used in the detectors or surrounding areas.

Analytical methods of analysis are also applied, like the Atomic Absorption Spectrometer (AAS) and the Inductively Coupled Plasma Mass Spectroscopy (ICP-MS). Both methods require pre-treating of the samples. In the case of the AAS, the wavelengths of light absorbed by the different elements are used to evaluate their concentrations in the solutions, and a good sensitivity (at ppb, sub-ppb level) can be reached, while an even better sensitivity at ppb-ppm and ppq-ppt level can be obtained with the ICP-MS. In this technique, the mass-to-charge ratio $(\mathrm{m} / \mathrm{e})$ is used to identify the isotopes of an element. Each peak in the mass spectrum has an intensity proportional to the isotope concentration. However, only a final low background measurement can assure the highest sensitivity to the identification of the internal contaminants in the crystal, and it is essential to collect the information for the full assembled detector configuration.

The presence of a wide range [15] of radioactive nuclides has been explored in inorganic crystal scintillators. When made for low-background purposes, like $\mathrm{NaI}(\mathrm{Tl}), \mathrm{CaF}_{2}(\mathrm{Eu})$, $\mathrm{CsI}(\mathrm{Tl}), \mathrm{ZnWO}_{4}, \mathrm{CdWO}_{4}$, and $\mathrm{Li}_{2} \mathrm{MoO}_{4}$, they can have standard contaminants in the range $0.01-1 \mathrm{mBq} / \mathrm{kg}$.

The initial impurity concentrations in the used materials, the used processes, and the handling protocols contribute to determine the achievable levels of radiopurity. The tools and abrasives used for crystals' cutting and polishing should be carefully selected. In particular, after the ingots production, all the materials used for the detector production and assembly have to be selected for radiopurity and their number should be minimised. Moreover, all the operations on the bare crystals require selected and controlled environment and procedures, in order to avoid possible surface pollution (see, e.g., Refs. [15,16]).

Nevertheless, slight differences in the residual contaminants of detectors may still arise, even if the same procedures are applied. In fact, some casual pollutions during the growth and handling procedures may in principle be possible, due to the industrial environment of the production sites. Differences may also depend on the selected part 
of the boule/ingot used to build the detector (a possible non-uniformity of contaminant distribution can play a role [17]).

In addition, during assembly, transport, storage, installation, etc., further pollution may also occur (e.g., at any step there can be the danger of radon contamination, if there is a contact with atmospheric air; the road-transport is preferred, in the shorter time possible, to minimize the cosmogenic activation; the installation is performed in HP nitrogen atmosphere, etc.). However, if accurate protocols are followed, all the critical aspects can be reduced, and the reduction of contaminants and the optimization of the detector performances can be achieved.

Focusing on DM investigation, a major effort has been done for the R\&D of $\mathrm{NaI}(\mathrm{Tl})$ crystal scintillators. A first advantage of $\mathrm{NaI}(\mathrm{Tl})$ is the absence of long lived isotopes. Other fundamental aspects to be considered are the method of crystallisation, the purification of the powders, the specific production line, the ovens, the crucible and so on. The growth of ULB NaI(Tl) crystals was made by the Kyropoulos method using selected platinum crucibles, with dedicated protocols [18]. In case of ULB NaI(Tl) crystals, it does not seem useful to increase the numbers of re-crystallisations to improve radiopurity, whereas specific actions on the chemical/physical purifications of the powders are performed. As an example, in the case of DAMA/LIBRA, the final residual contaminants in the powders of the ULB NaI(Tl) were for ${ }^{238} \mathrm{U}: 0.02 \mathrm{ppb}$, for ${ }^{232} \mathrm{Th}: 0.02 \mathrm{ppb}$, and for ${ }^{n a t} \mathrm{~K}:<0.1 \mathrm{ppm}$ ( $95 \%$ C.L.), respectively, by MS and AAS measurements. Even if the Tl concentration in the detectors is $\simeq 0.1 \%$, the TII powders were specifically selected and dedicated chemi$\mathrm{cal} / \mathrm{physical}$ purifications were applied. The residual contaminants were for ${ }^{238} \mathrm{U}: 0.8 \mathrm{ppb}$, for ${ }^{232} \mathrm{Th}: 1.2 \mathrm{ppb}$, and for ${ }^{n a t} \mathrm{~K}:<0.06 \mathrm{ppm}$ (95\% C.L.), respectively, by MS and AAS measurements. The Kyropoulos crystallisation process (platinum crucibles are preferred) by itself represents a further important purification step of the residual contamination in the powders. In fact, the residual background directly measured underground with the DAMA crystals used as detectors is at ppt level for all three radionuclides ${ }^{238} \mathrm{U},{ }^{232} \mathrm{Th}$ and ${ }^{40} \mathrm{~K}$ [18].

\section{DAMA/LIBRA Investigation on DM with NaI(Tl) Detectors}

In the DAMA/LIBRA experiment, $\mathrm{NaI}(\mathrm{Tl})$ crystals are employed as scintillator detectors to investigate the presence of DM particles in the Milky Way. For this purpose, the DM-AM signature is exploited. This signature is due to the revolution velocity of the Earth around the Sun which is in movement with respect to the galactic frame. The composition of the velocity of the Earth and of the Sun in the galactic frame results in the modulation of the flux of DM particles crossing the Earth. In particular, the flux is expected to be larger around $\simeq 2$ June, when the two velocities are aligned, while it is expected to be smaller around $\simeq 2$ December when the two velocities are opposite. Slight variations may occur in case of the presence of non-thermalized DM components in the galactic halo.

\subsection{The Target Material and the Experimental Set-Up}

The highly radiopure $\mathrm{NaI}(\mathrm{Tl})$ scintillators are a very good target detector to investigate the DM particle component in the galactic halo. They have many advantages:

1. well known technology;

2. high radiopurity level that can be obtained by material selections, the design of growth and handling protocols, by applying chemical/physical purification procedures, etc.;

3. crystal of large mass can be grown;

4. the experimental duty cycle is very high;

5. it is possible to perform routine calibrations in the keV energy range without perturbing the data taking;

6. the operational conditions can be controlled and stabilized;

7. no microphonic noise is present;

8. the discrimination between scintillation signal and noise (mainly photomultiplier (PMT) noise, Cherenkov light in the light guides and in the PMT windows, and afterglows) close to the energy threshold is effective thanks to: the high number of 
photoelectrons $/ \mathrm{keV}$, the different time decay of PMT noise pulses (of the order of tens of ns) and of the scintillation pulses (of the order of hundreds of ns);

9. high light yield ( $\sim 40,000$ photons $/ \mathrm{MeV})$ that allows for having a threshold at keV range;

10. pulse shape discrimination feasible at a reasonable level when of interest [19];

11. no re-purification or cooling down/warming up procedures needed (implying high reproducibility, high stability, etc.);

12. it is possible to have a multi-detectors set-up that can have an important role for DM investigations and for background recognition;

13. sensitivity to both high (by iodine target) and low (by Na target) mass DM candidates;

14. high sensitivity to a DM particles' candidate with spin-independent, spin-dependent and mixed couplings;

15. high sensitivity to several other existing scenarios and to many other possible candidates including those producing just electromagnetic radiation in the interaction;

16. effective to investigate the DM-AM signature by considering all its characteristics;

17. "ecologically clean" apparatus, having no safety issues;

18. more affordable than other detectors considered in the field;

19. compact in size, requiring small occupancy in an underground laboratory;

The DAMA/LIBRA experiment can be considered as the successor of the pioneer DAMA/NaI set-up, and it has been taking data in two configurations; the first one was DAMA/LIBRA-phase1 and the second, presently running, is DAMA/LIBRA-phase2 [20]. DAMA/LIBRA-phase2 is an improved version of the experiment in which, as a main upgrade, all the PMTs were changed to be replaced with the Hamamatsu R6233MOD PMTs; these devices have higher quantum efficiency and lower background with respect to those used in phase1 [21,22]. The upgrade has been performed in 2011 and the goal to reach a software energy threshold at $1 \mathrm{keV}$ was achieved.

DAMA/LIBRA-phase2 set-up is made of 25 highly radio-pure $\mathrm{NaI}(\mathrm{Tl})$ crystal scintillators (5-rows by 5-columns matrix) having $9.70 \mathrm{~kg}$ mass each one; the residual contaminants of all the parts of the apparatus have been measured in details and they are reported in Ref. [18]. The schematic view of the building hosting the DAMA/LIBRA apparatus and of its shield is shown in Figure 1.
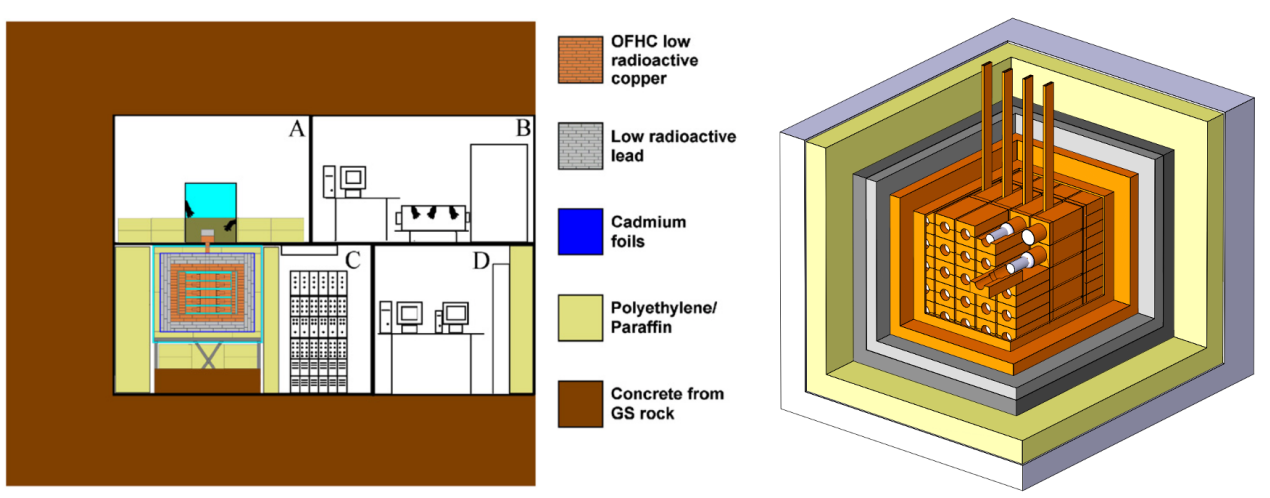

Figure 1. Left: schematic view of the building hosting the DAMA/LIBRA apparatus (not to scale). About $1 \mathrm{~m}$ concrete (made from the Gran Sasso rock material) almost fully surrounds (mostly outside the building) the apparatus. In particular, the room A hosts the glove-box for calibrations; some facilities are in the room $B$; the sensitive parts of the apparatus are in the room $C$ inside the multicomponent shield as well as the electronics; the computer for DAQ is in the room D. Right: schematic view of the passive shield of the DAMA/LIBRA apparatus. Reprinted with permission from [18]. Copyright 2008 Elsevier.

Two 10-cm long UV light guides of Suprasil B quartz are coupled to the detectors on the two end faces of the crystal; they connect each crystal to the low background PMTs working in coincidence at a single photoelectron level. The detectors are placed inside a sealed low-radioactive copper box installed in the center of a low-radioactive $\mathrm{Cu} / \mathrm{Pb} / \mathrm{Cd}$ - 
foils/polyethylene/paraffin shield. About $1 \mathrm{~m}$ concrete, obtained from the Gran Sasso rock material, is present outside the detector barrack and completely encloses it. The concrete layer acts as an external shield to moderate neutrons. A layer of neoprene lies down on the floor of laboratory to separate it from the concrete basement. A thickness of several tens of $\mathrm{cm}$ of paraffin fills the space between the basement and the metallic structure.

To isolate the set-up from the environmental air of the underground laboratory, a threefold-level sealing system is in use. In addition, the set-up is maintained in HP (highpurity) nitrogen atmosphere. To assure the stability of the temperature, two independent and redundant systems of air conditioning are active. The huge heat capacity of the multitons passive shield $\left(\approx 10^{6} \mathrm{cal} /{ }^{\circ} \mathrm{C}\right)$ guarantees further stability of the detectors' operating temperature. The operating conditions are monitored continuously with a dedicate data acquisition system connected to several probes and sensors. The running conditions are very stable at a level better than $1 \%$ (see, e.g., Ref. [23]).

The light yield of the detectors in the phase 2 ranges from 6 to 10 photoelectrons $/ \mathrm{keV}$. To calibrate the energy scale of the detectors, various $X$-rays $/ \gamma$ sources are used. The calibration operations are carried out without any change of the experimental conditions (for details see, e.g., Ref. [18]). In addition, some gamma lines due to the presence of residual radioactive contamination are also considered. As an example, the ${ }^{40} \mathrm{~K}$, present in the crystal at ppt level, produces double coincidence events due to internal X-rays emission after its EC decay. A line at $3.2 \mathrm{keV}$ is close to the software energy threshold. The DAQ system records both single-hit events (where just one of the detectors fires) and multiple-hit events (where more than one detector fires) up to the $\mathrm{MeV}$ region, although the trigger optimization is performed for the lowest energy events.

All the analysis to estimate the radio-purity of the experiments are described e.g., in Refs. $[4-6,18,22,24]$ and references therein while a detailed description of the DAMA/LIBRA set-up is reported in Refs. [4-6,18,24].

The DM-AM signature is a very powerful signature because the expected features of the related signal must satisfy several conditions: the rate has a component modulated according to a cosine function (1), the period of the modulation is one year (2), and its phase is $\simeq 2$ June (3); this modulation should be present in the keV energy range considering the energy released by the DM particle in the interaction with target detectors (4); the modulated signal is characterized by events in which just one detector "fires" (single-hit events), since the interaction of a DM particle with more than one detector has a negligible probability (5); in the region where the signal is maximum, the modulation amplitude is expected to be $\simeq 7 \%$ when Maxwellian-like halo distributions are considered, but it can be larger (even up to $\simeq 30 \%$ ) in some scenarios [25-29] (6). The NaI(Tl) target detectors are sensitive to many DM candidates, interaction types, and scenarios (see, e.g., Ref. [30] and references therein). A possible fake effect or systematic should not only account for the measured modulation amplitude but in addition simultaneously satisfy all the above-mentioned conditions (see, e.g., Ref. [30] and references therein).

\subsection{The Model-Independent Results on DM-AM}

Six full annual cycles of DAMA/LIBRA-phase2 have been considered in the analysis of the DM-AM signature; the whole exposure is 1.13 ton $\times y$, while including also DAMA/NaI and DAMA/LIBRA-phase1, the total exposure is 2.46 ton $\times$ yr. The same procedures already adopted for the DAMA/LIBRA-phase1 [4-6,18,24] have also been exploited in the analysis of DAMA/LIBRA-phase2 [7,23,31].

Many analyses have been performed on the data to study the peculiarities expected from the DM-AM signature. In this publication, a part of the results and examples will be reported. Additional, detailed results have been presented in Refs. [7,23,31]. As an example, in Figure 2, the residual rates of the single-hit scintillation events measured in the former DAMA/LIBRA-phase1 and the new DAMA/LIBRA-phase2 are reported. As described e.g., in Refs. [3-6,24,32], the residual rates are calculated from the rate of the single-hit events by subtracting the unmodulated part, in the energy interval from the software 
energy threshold of DAMA/LIBRA-phase1 (2 keV) to $6 \mathrm{keV}$. The null hypothesis of the absence of modulation is discarded by $\chi^{2}$ test at very high C.L.: $\chi^{2} /$ d.o.f. $=199.3 / 102$, and related $p$-value $=2.9 \times 10^{-8}$. The residual rates for the lower energy threshold of $1 \mathrm{keV}$ are shown elsewhere $[7,23,31]$.

The residual rates of the single-hit events for the full data sets DAMA/NaI, DAMA/ LIBRA-phase1, and DAMA/LIBRA-phase2 was fitted by the function (see Figure 2): $A \cos \omega\left(t-t_{0}\right)$, where the period $T=\frac{2 \pi}{\omega}=1 \mathrm{yr}$ and the phase $t_{0}=152.5$ day (2nd June) are those expected by the DM-AM signature. The fitted modulation amplitude is $A=$ $(0.0102 \pm 0.0008) \mathrm{cpd} / \mathrm{kg} / \mathrm{keV}\left(\chi^{2} /\right.$ d.o.f. $\left.=113.8 / 138\right)[7]$.

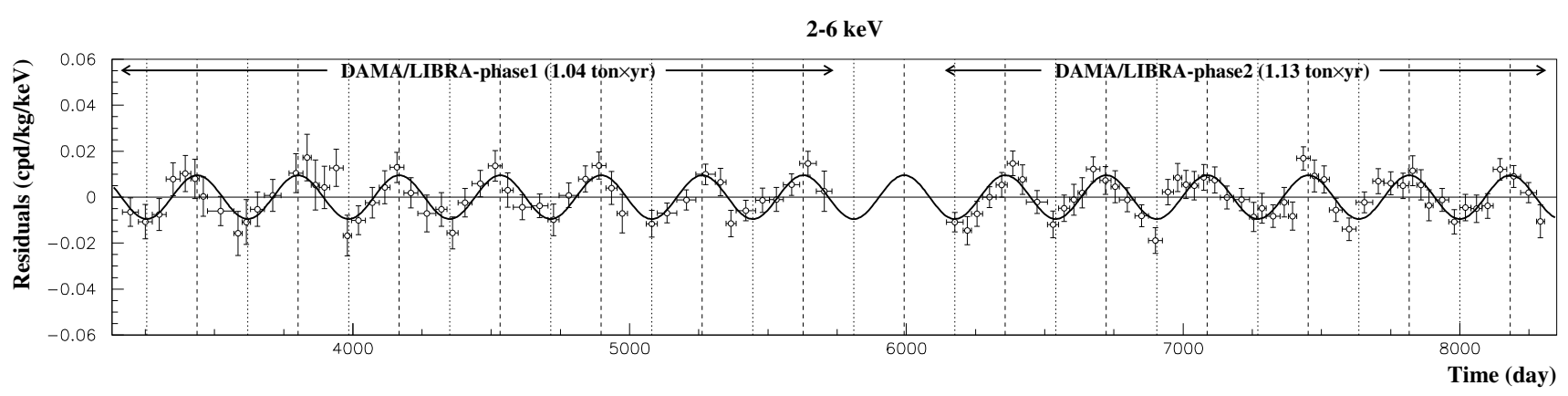

Figure 2. Residual rate for single-hit scintillation events in the (2-6) keV energy interval in DAMA/LIBRA-phase1 and DAMA/LIBRA-phase2. The shown curve is the cosinusoidal function $A \cos \omega\left(t-t_{0}\right)$ with period $T=\frac{2 \pi}{\omega}=1 \mathrm{yr}$, phase $t_{0}=152.5$ day (2nd June) and modulation amplitude, $A$, equal to the central value obtained by best fit on the data points of DAMA/LIBRA-phase1 and DAMA/LIBRA-phase2. Other residual rates for different energy regions and, in particular, with lower energy threshold of $1 \mathrm{keV}$ are reported in Refs. [7,23,31]. Reprinted with permission from [31]. Creative Commons License CC BY 4.0.

If the period and the phase of the signal are kept free, fitting the full exposure ( 2.46 ton $\times$ yr), the result of the fit is: the modulation amplitude of the single-hit scintillation events $A=(0.0103 \pm 0.0008) \mathrm{cpd} / \mathrm{kg} / \mathrm{keV}$, the period $T=(0.999 \pm 0.001) \mathrm{yr}$, and the phase $(145 \pm 5)$ days [7], all these values are well in agreement with those expected for DM particles; the C.L. is $12.9 \sigma$.

This result confirms the evidence of a signal satisfying all the requirements of the model independent DM-AM signature. Neither systematics nor side reactions able to mimic the DM-AM signature (i.e., to account for the measured modulation amplitude and to simultaneously satisfy all the requirements of the signature) have been found so far.

Other analyses, not shown here, exclude the presence of any background modulation in the energy regions above $6 \mathrm{keV}$. Among them, the time behaviour of the integral rate above $90 \mathrm{keV}, \mathrm{R}_{90}$, has been analysed [6,7,24,30]; similar conclusions have also been obtained considering data in other energy regions.

Among other performed analyses, a further investigation on DAMA/LIBRA—phase2 data was done-as in the previous DAMA/NaI and DAMA/LIBRA-phase1 experiments $[4-6,24,32]$ - in the residual rate of the multiple-hit events. These events are collected and analyzed through the same hardware and software procedures as for the single-hit ones. Because of the negligible probability that a DM particle interacts in more than one detector, a DM signal is expected only in the single-hit residual rate. Hence, the proper comparison of single-hit and multiple-hit residuals is equivalent to compare the cases of DM particles beam-on and beam-off. This implies a further test on the background in the same energy region where the positive effect is observed.

The residual rates of the single-hit scintillation events and of the multiple-hit events, as collected in a single cycle, are reported in Figure 3 for DAMA/LIBRA-phase2; the energy range is (1-6) $\mathrm{keV}$. The single-hit residual rate shows a clear modulation, satisfying all the peculiarities of the DM-AM signature, while the multiple-hit one does not; the fitted modulation amplitude for the multiple-hit residual rate is: $(0.0004 \pm 0.0004) \mathrm{cpd} / \mathrm{kg} / \mathrm{keV}$, well compatible with zero. Therefore, evidence of annual modulation with the proper 
peculiarities of the DM-AM signature is present in the single-hit residuals (class of events to which the DM events belong), and it is absent in the multiple-hit residuals (class of events to which only background events belong).

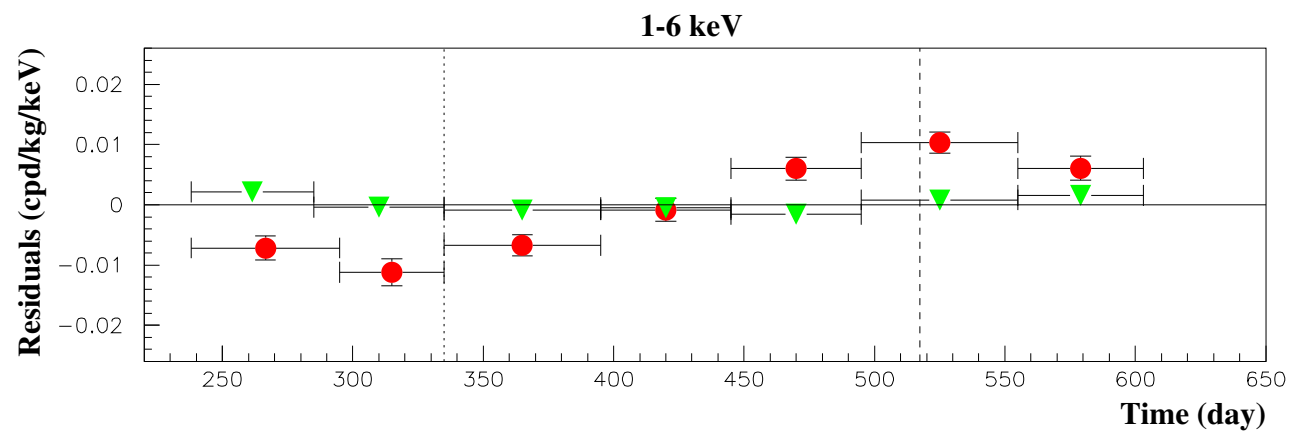

Figure 3. Residual rates of single-hit events (circles), class of events to which DM events belong, and of multiple-hit events (filled triangles), class of events to which DM events do not belong. The DAMA/LIBRA-phase2 is considered; the data points are reported as collected in a single annual cycle and have been obtained through the same hardware and software procedures. The time scale is the same as the previous DAMA papers for consistency $[4-6,24,32]$. The experimental points present the errors as vertical bars and the widths of the associated time bins as horizontal bars. Reprinted with permission from [31]. Creative Commons License CC BY 4.0.

The annual modulation reported at low energy can also be pointed out through the maximum likelihood method (fixing $T=1 \mathrm{yr}$ and $t_{0}=152.5$ day) by investigating the modulation amplitude, $S_{m}$, as a function of energy. The modulation amplitudes of the whole data sets: DAMA/NaI, DAMA/LIBRA-phase1 and DAMA/LIBRA-phase2 (total exposure 2.46 ton $\times$ yr) are reported in Figure 4. Because of the lower energy threshold, the data points below $2 \mathrm{keV}$ belong only to DAMA/LIBRA-phase2. A positive signal is present in the (1-6) keV energy range, confirming the previous analyses. $S_{m}$ data points compatible with zero are present above $6 \mathrm{keV}$; for example, the test of the hypothesis that the $S_{m}$ values have random fluctuations around zero in the (6-14) keV energy interval gives $\chi^{2}=19.0$ for 16 d.o.f., and the upper tail probability is $27 \%$. In the (6-20) keV energy interval, the $\chi^{2} /$ d.o.f. is $42.6 / 28$, which corresponds to the upper tail probability of $4 \%$. This rather large $\chi^{2}$ is mainly due to two data points far away from the (1-6) keV energy interval: their centroids are at 16.75 and $18.25 \mathrm{keV}$. Excluding only the first and either the two points the $p$-values are $11 \%$ and $25 \%$, respectively.

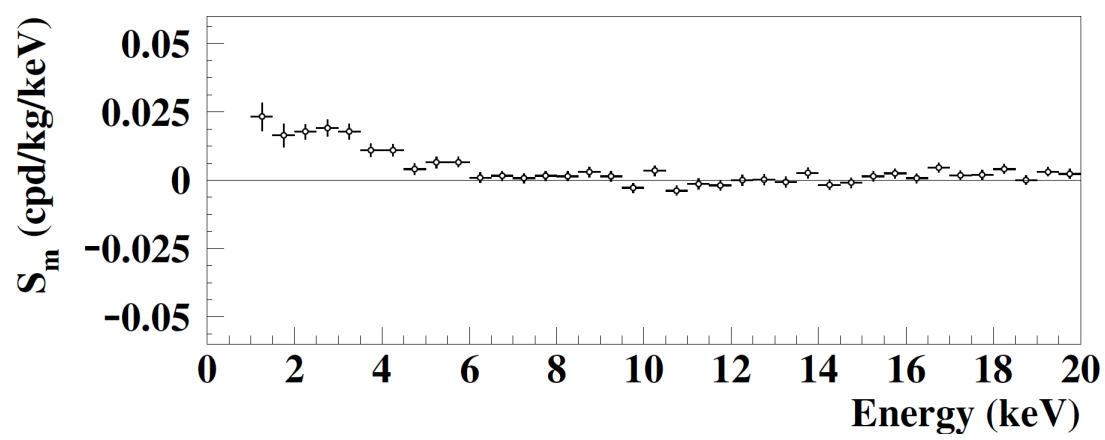

Figure 4. Modulation amplitudes, $S_{m}$, for DAMA/NaI, DAMA/LIBRA-phase1 and DAMA/LIBRAphase2 (total exposure 2.46 ton $\times \mathrm{yr}$ ). The data points below $2 \mathrm{keV}$ belong only to DAMA/LIBRAphase2. The energy bin $\Delta E$ is $0.5 \mathrm{keV}$. The modulation is evident for the lowest energy data points, and $S_{m}$ 's compatible with zero are present elsewhere. See text for comments. Reprinted with permission from [31]. Creative Commons License CC BY 4.0.

The observed annual modulation effect is well distributed in all the 25 detectors; see Refs. [4-6,24,31,33] for other details. 
No possible source of systematics or side reactions have been found; therefore, cautious upper limits have been set which are not able to account for the modulation amplitudes measured by DAMA, and are not able to simultaneously satisfy all the requirements of the DM-AM signature; for details, see Refs. [3-6,32,34-36]. In particular, we recall thaton the contrary to what was claimed in Ref. [37] and sometimes uncritically repeated by some others-no possibility exists for any appreciable contribution from the combination of solar neutrinos and muons (one with a phase much lower and the other larger than the one measured by DAMA experiments) for the many scientific quantitative reasons reported in Ref. [34] and Refs. therein. As evident, the DAMA/LIBRA multi-detectors target mass, $\simeq 250 \mathrm{~kg}$ in total, cannot detect any significant contribution to the modulation of its measured single-hit events from those sources, which are typically investigated underground by multi-hundred-tons experiments. Similar analyses on systematics and side reactions were also performed for the DAMA/NaI data [3,32].

In conclusion, the long-standing annual-modulation evidence observed by DAMA is model-independent, and it can be compatible with a wide set of DM scenarios as discussed in DAMA (see, e.g., Refs. [8,30]) and other literature.

\subsection{Perspectives}

To efficiently disentangle some of the numerous potential candidates and theoretical frameworks, decreasing the DAMA/LIBRA energy threshold below $1 \mathrm{keV}$ is important. In particular, the experimental sensitivity to the DM-AM is connected to the product: $\epsilon \times \Delta E \times M \times T \times\left(\alpha-\beta^{2}\right)$, where $\epsilon$ is the overall efficiency, $\Delta E$ is the energy region where the DM-AM is present, $M$ is the exposed mass, $T$ is the running time, and $\left(\alpha-\beta^{2}\right)$ term gives information about the distribution of the collected data along each annual cycle. In particular, $\alpha=\left\langle\cos ^{2} \omega\left(t-t_{0}\right)\right\rangle$ is the mean value of the squared cosine and $\beta=\left\langle\cos \omega\left(t-t_{0}\right)\right\rangle$ is the mean value of the cosine (the averages are taken over the lifetime of the data taking and $t_{0}=152.5$ day, i.e., 2 nd June); thus, $\left(\alpha-\beta^{2}\right)$ indicates the variance of the cosine (i.e., it is 0.5 for a full year of data taking). This quantity is relevant to pursue the DM-AM investigation. Lowering the software energy threshold corresponds to increasing the acceptance efficiency. An improved experimental sensitivity can investigate the DMAM more extensively and improve the modulation parameters' measurement, e.g., the phase, which yields relevant information. Indeed, the DAMA project's goal was to point out model-independent evidence for the presence of DM particles in the galactic halo and study the characteristics of such particles and related astrophysical, nuclear and particle physics' scenarios. In particular, a few primary purposes of achieving a lower software energy threshold are:

- to explore the DM-AM at lower software energy threshold, with high overall efficiency, to untangle among some of the many proposed theoretical frameworks;

- to investigate, also considering the recent GAIA measurements, feasible presence of streams (as we already did for Sagittarius in the past), and caustics in the Galaxy, including Sun's effects gravitational focusing;

- to examine the nuclear parameters assumed in model-dependent corollary analyses;

- to investigate the diurnal modulation with an increased sensitivity and other feasible diurnal effects due for e.g., to the Earth shadow and the channelling (refer to the DAMA literature for a complete discussion);

- to study rare processes other than DM, such as analyzing either other regions of the energy spectrum or studying specific peculiarities of the processes investigated, as performed earlier and published using the DAMA/NaI and DAMA/LIBRA-phase1 data (from $\mathrm{keV}$ up to tens $\mathrm{MeV}$; see Refs. [38-48]).

The DAMA collaboration has been operating towards this objective. The purpose is to further improve the signal-to-noise ratio approaching the software energy threshold to untangle the noise pulses (time decay of order of tens ns) and the scintillation events (time decay of the order of $240 \mathrm{~ns}$ ), including a high overall efficiency, below one keV [18,21]. Thus, new high Q.E. and low radioactivity PMTs have been developed by the Hamamatsu 
Co. based on the DAMA requirements, e.g.,: (i) Q.E. at $\lambda=420 \mathrm{~nm} \mathrm{30-40 \% ;} \mathrm{(ii)} \mathrm{dark}$ current $<100$ cps; (iii) 3-inch window diameter; (iv) specific shape and size to fit the already-existing honeycomb-shaped copper shield around PMTs and to minimize the amount of material; (v) multiplication factor $>10^{6}$; (vi) peak/valley ratio $>2.5$; (vii) PMTs radio-purity at the level of a few $\mathrm{mBq} / \mathrm{PMT}$.

Moreover, to improve the signal/noise ratio, a new optimized preamplifier-voltage divider system, integrated on the same Pyralux board, was developed and mounted on the new metal PMTs. The ultra low noise operational amplifier LMH6624, developed by Texas Instruments, is the core of the new preamplifier produced. It works at $\pm 5 \mathrm{~V}$, with an input bias current of $-15 \mu \mathrm{A}$ at $300 \mathrm{~K}$ and a bandwidth of $1.5 \mathrm{GHz}$. A view of this new optimized preamplifier-voltage divider system is shown in Figure 5.

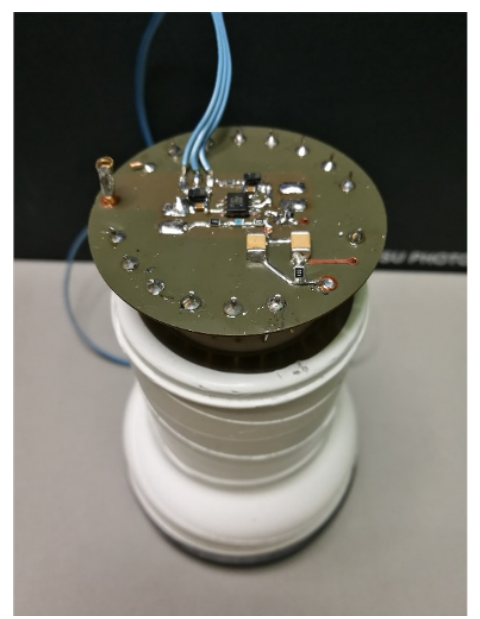

Figure 5. View of the new metal R11065-20 MOD PMT with the preamplifier-voltage divider system.

The preamplifier-voltage divider system is printed on the first and third layer of a Pyralux board; between these two layers, a ground layer is deposited. Such a system is installed on the PMT backside in the shield's copper honeycomb structure. Thus, the preamplifiers are as close as possible to the input source (the anode of the PMT) instead of those used so far in DAMA/LIBRA-phase2, which are installed beyond the inner part of the set-up's shield. The upgrade is now possible considering the improved radiopurity of the new metallic PMTs and the novel miniaturized preamplifier-voltage divider system. In summary, a larger signal/noise factor can be achieved with respect to an external preamplifier and the overall radio-purity is improved.

Analyses with and without the preamplifier have been performed to characterize the metallic PMTs, their response, and the preamplifier-voltage divider system's performance:

- $\quad$ Spectral response of the PMT, expressed by the Q.E., as a function of $\lambda$;

- $\quad$ Single Photon Pulse Height Distribution of the metallic PMTs obtained by irradiating the device with single photon pulse;

- Dark Pulse Height Distribution of the PMTs;

- Dark Current.

The preamplifier-voltage divider system (overall mass $14.1 \mathrm{~g}$ ) has been measured in an HP-Ge detector in the STELLA facility (at the LNGS) during 6.87 days to test its residual radioactivity. In particular, the residual activity of ${ }^{232} \mathrm{Th},{ }^{235} \mathrm{U},{ }^{40} \mathrm{~K},{ }^{137} \mathrm{Cs}$, and ${ }^{60} \mathrm{Co}$ is much lower than that of a single photomultiplier. Only the ${ }^{226} \mathrm{Ra}$ activity is slightly higher, at the level of a $13 \mathrm{mBq}$ per preamplifier-voltage divider system. However, this is a common characteristic for such kind of equipment: the used Pyralux material usually has an activity of about hundred times lower for ${ }^{226} \mathrm{Ra}$ and about 30 times lower for ${ }^{228} \mathrm{Ra}$ and ${ }^{228} \mathrm{Th}$. Nevertheless, the small activity is principally due to the electronic elements. However, the residual total background due to the preamplifier-voltage divider system is negligible because it is placed behind the PMT and shielded largely by a honeycomb $\mathrm{Cu}$ structure. 
Four DAMA/LIBRA detectors are currently equipped and in data taking mode using the new metal R11065-20 MOD PMTs and the innovative preamplifier-voltage divider system. Other alternatives and cheaper configurations are under study to lower the detectors' software energy threshold in light of these developments and goals. The work is in progress also considering the use of new Transient Digitizers, and a thorough analysis is being carried out; results will be published.

\section{Investigating the Directionality of DM with $\mathrm{ZnWO}_{4}$ Crystal Scintillators}

Another effective approach, in the case of DM candidate particles that induce nuclear recoils, is the directionality technique. The directionality investigates the correlation between the direction of the nuclear recoils induced by DM particles and the motion of the Earth in the galactic rest frame. In fact, due to the solar motion in the DM halo, a wind of DM particles is expected on the Earth with an apparently opposite direction with respect to the Earth velocity in the galactic rest frame. As a consequence of the Earth's rotation, an observer on the Earth's surface experiences a change in the DM average arrival direction during a sidereal day. The induced nuclear recoils' directions are expected to be strongly correlated with that of the DM particles. Thus, the study of the nuclear recoils directions can highlight the presence of a very distinctive signal, as expected for a large class of DM candidates interacting via elastic-scattering on target nuclei. On the other hand, the nuclear recoil range is very short: typically of the order of $\mu \mathrm{m}$ in solid detectors and of the order of a few $\mathrm{mm}$ in low pressure Time Projection Chambers. This makes the direct measurement of the recoil tracks very difficult. However, this limitation can be overcome by using anisotropic scintillation detectors [49-51].

In anisotropic scintillators, the detector response to heavy particles depends on their impinging direction with respect to crystal axes. Therefore, in the case of nuclear recoils induced by DM particles, the measured energy spectrum at low energy is expected to change its shape during the sidereal day as a consequence of the changing orientation of the crystal axes with respect to the DM wind. This effect can be pointed out as a peculiar variation of the counting rate measured in a given low energy window and offers the possibility to highlight the DM signal with respect to the electromagnetic background (for details, see, e.g., in Refs. [10,50,51]).

The potential offered by the anisotropic properties of $\mathrm{ZnWO}_{4}$ crystal scintillator in the search for DM with the directionality approach was indicated for the first time in Ref. [49]. Recent measurements and R\&D show that the $\mathrm{ZnWO}_{4}$ detector can offer suitable features for the purpose $[10-12,52,53]$. The light output and the pulse shape indeed depend on the particle direction with respect to crystal axes in the case of heavy particles and nuclear recoils, while no difference is observed for $\gamma / \beta$ radiation [49]. The $\mathrm{ZnWO}_{4}$ detector also ensures a high atomic weight and the possibility to obtain large crystals with masses of some $\mathrm{kg}$ [54]. Moreover, the three target nuclei, $\mathrm{Zn}, \mathrm{W}$ and $\mathrm{O}$, have very different masses and make the detector sensitive to DM candidates with small and large mass. In light of this, the ADAMO project was considered and R\&D has been progressing [10-12]. DAMA and the Institute for Nuclear Research of NASU (Ukraine) have developed various $\mathrm{ZnWO}_{4}$ detectors $[17,52,53,55,56])$, and an R\&D activity is presently ongoing with the Nikolaev Institute of Inorganic Chemistry (Novosibirsk, Russia). Large $\mathrm{ZnWO}_{4}$ detectors, produced with the conventional and the low-thermal gradient (LTG) Czochralski technique in a platinum crucible [54], have been measured underground at LNGS in the DAMA/R\&D set-up $[52,53,55-57]$.

$\mathrm{ZnWO}_{4}$ scintillators with very good radiopurity levels have recently been developed. In particular, the measured upper limits on contaminants activities are: $20 \mu \mathrm{Bq} / \mathrm{kg}$ for ${ }^{40} \mathrm{~K}$, $2 \mu \mathrm{Bq} / \mathrm{kg}$ for ${ }^{226} \mathrm{Ra}$, and in the range $(0.17-1.3) \mu \mathrm{Bq} / \mathrm{kg}$ for ${ }^{228} \mathrm{Th}[11,52]$.

The measurements performed at LNGS also confirmed that the $\mathrm{ZnWO}_{4}$ light output at room temperature is about $20 \%$ of the $\mathrm{NaI}(\mathrm{Tl})$ scintillator. Previous studies have shown that it is possible to improve the light output of the crystal when working at low temperature [58]. This feature will also be verified at LNGS, where a small cryostat is under 
testing for implementing and optimizing the cooling system. This system will allow the measurement of the light output at a stable working temperature of about $-50{ }^{\circ} \mathrm{C}$.

New measurements, with important implications for the use of $\mathrm{ZnWO}_{4}$ as a detector for the DM directionality approach, have been recently performed by the ADAMO project using a $(10 \times 10 \times 10.4) \mathrm{mm}^{3}, 7.99 \mathrm{~g}$ mass, crystal scintillator [12]. In the measurements performed with a neutron generator, the small size of the crystal reduces the probability of multiple scattering of the neutron in the detector. The used $\mathrm{ZnWO}_{4}$ is a second crystallization crystal produced with the LTG Czochralski technique from zinc tungstate crystals made from tungsten oxide additionally purified by double sublimation of tungsten chlorides [59].

The first measurement, carried out at LNGS, allowed the study of the detector response to $\alpha$ particles and $\gamma$ quanta, and the measurement of the $\alpha / \gamma$ ratio. The $\alpha / \gamma$ ratio and, more generally, the quenching factor is the ratio between the detected energy in the energy scale calibrated with $\gamma$ sources to the energy of the heavy ionizing particle. The $\mathrm{ZnWO}_{4}$ crystal was coupled to a Hamamatsu H11934-200 PMT (Ultra Bialkali photocathode with quantum efficiency $\simeq 43 \%$ at $400 \mathrm{~nm}$ and $\simeq 25 \%$ at $500 \mathrm{~nm}$ ). The scintillation profiles were acquired with a LeCroy WaveSurf24X-sA oscilloscope (2.5 GSamples/s, $200 \mathrm{MHz}$ ) using a time window of $100 \mu \mathrm{s}$. A collimated beam of $\alpha$ particles produced by an ${ }^{241} \mathrm{Am}$ source and mylar absorbers with different thickness were used to repeat the measurement at different $\alpha$ energies. The impinging $\alpha$ energies were preliminarily measured with a CANBERRA Alpha Spectrometer model 7401VR. For each measurement with the $\mathrm{ZnWO}_{4}$ detector, the energy calibration was determined using ${ }^{22} \mathrm{Na}$ and ${ }^{137} \mathrm{Cs} \gamma$ sources. The measured $\alpha / \gamma$ ratio as a function of the energy for the three directions of the $\alpha$ beam relatively to the crystal planes is reported in Figure 6 [12].

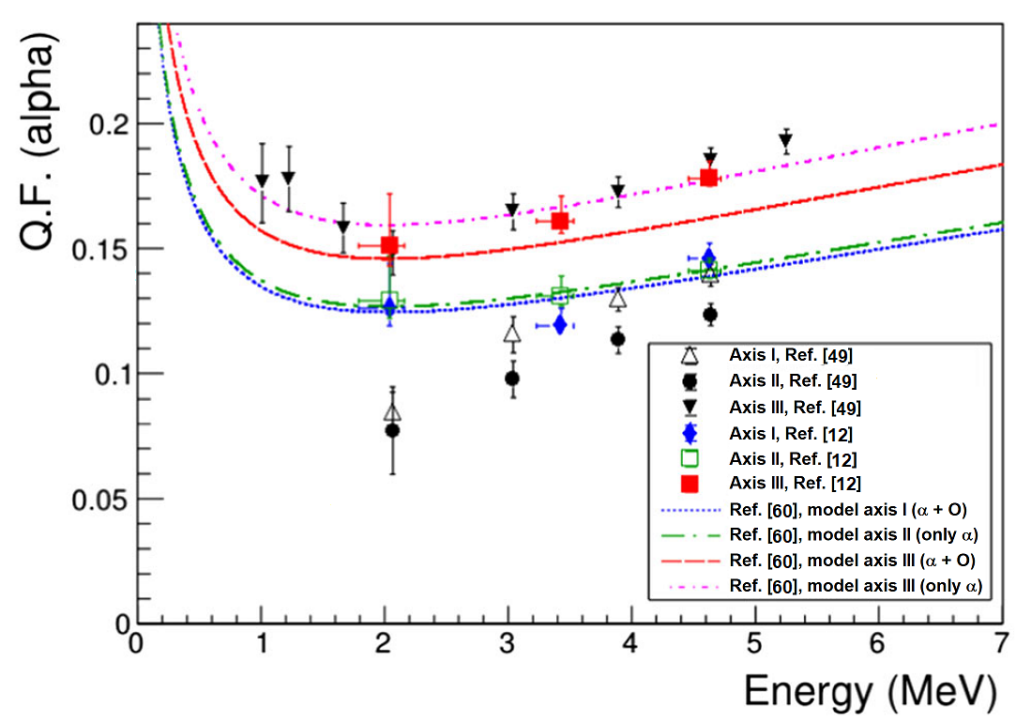

Figure 6. $\alpha / \gamma$ ratio measured with a $\mathrm{ZnWO}_{4}$ scintillator in Ref. [12] (colored points) for the three directions of the $\alpha$ beam relatively to the crystal planes and various energies. The values measured in Ref. [49] are also reported for comparison (black points). The anisotropic behaviour of the crystal is evident. The model of Ref. [60] is also reported for each crystal axis. It has been obtained by fitting the data from $\alpha$ s and from recoils or only from $\alpha$ s. Reprinted with permission from [12]. Copyright 2020 Springer Nature.

The data confirm the anisotropic features of $\mathrm{ZnWO}_{4}$ crystal scintillator in the case of $\alpha$ particles. In particular, the $\alpha / \gamma$ ratio measured along crystal axis III is about 1.2 times larger than that measured along the axes I and II, where the measured quenching factors are quite similar. The reported uncertainties are mainly due to the evaluation of the impinging alpha energy, which is slightly degraded in air. As shown in Figure 6, the measured quenching factors and anisotropic effect are compatible with those of Ref. [49]. The behaviour of the 
$\alpha / \gamma$ ratio as expected for each crystal axis in the model of Ref. [60], based on the semiempirical classical Birks formula [61], is also reported in Figure 6 (see Ref. [12] for details).

The second measurement was carried out at ENEA-Casaccia Laboratory. A schematic view of the experimental set-up is reported in Figure 7.

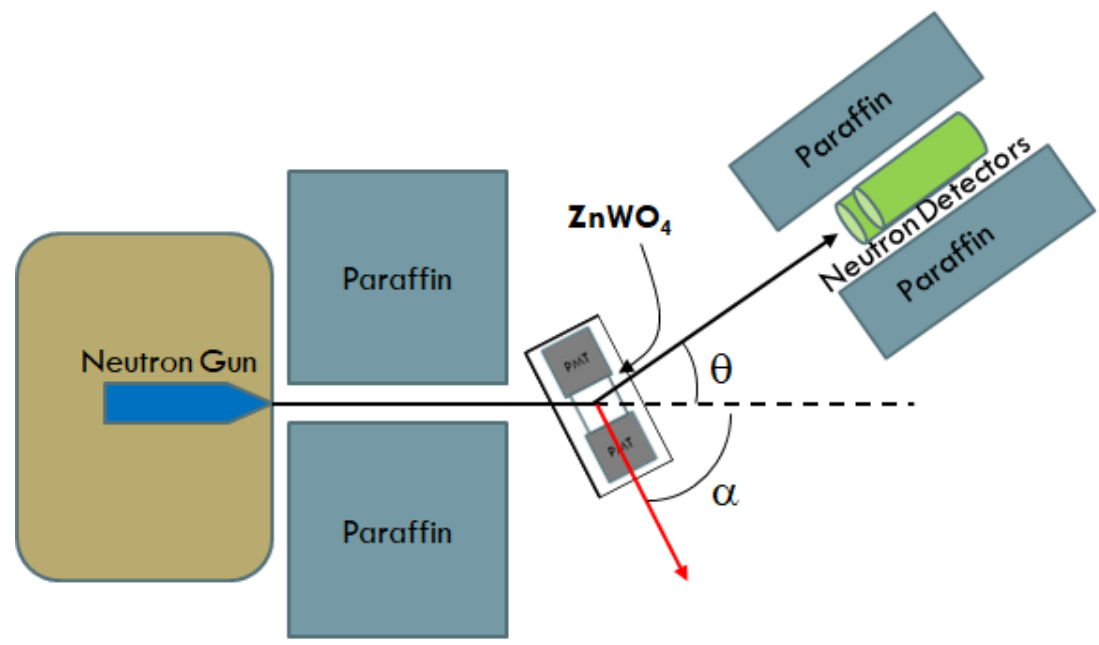

Figure 7. Schematic view from the top of the experimental set-up used at ENEA-Casaccia Laboratory for the measurements of the anisotropic response of the $\mathrm{ZnWO}_{4}$ detector in the case of nuclear recoils. The two neutron detectors are one above the other. See Ref. [12] for details. Reprinted with permission from [12]. Copyright 2020 Springer Nature.

Monochromatic neutrons with energy around $14.7 \mathrm{MeV}$ are generated with a Thermo Scientific portable generator MP 320 through the $d(t, \alpha) n$ reaction. After a single elastic scattering on a target nucleus, the recoiling nucleus is detected by the $\mathrm{ZnWO}_{4}$ detector, while the scattered neutron can be tagged by one of the two neutron detectors placed at a given scattering angle. When that happens, both the energy and the direction of the recoiling nucleus are determined by the selected scattering angle, while the detected energy in the $\mathrm{ZnWO}_{4}$ detector, measured in $\mathrm{keV}$ electron equivalent (keVee), allows the determination of the quenching factor. Therefore, the quenching factors for nuclear recoils along different crystallographic axes and for different recoil energies can be measured changing the $\mathrm{ZnWO}_{4}$ detector orientation and the neutron detectors position, respectively.

In this measurement, the $\mathrm{ZnWO}_{4}$ scintillator was optically coupled to two Hamamatsu H11934-200 PMTs; their signals were recorded by a CAEN DT5720 transient digitizer with 250 MSamples/s. The two neutron detectors, by Scionix, employ an EJ-309 liquid scintillator, which can discriminate neutrons interactions from the $\gamma$ background by an excellent Pulse Shape Discrimination (PSD) capability. The trigger was generated when a coincidence between the $\mathrm{ZnWO}_{4}$ detector and the EJ-309 detectors occurred within a time window of $\pm 500 \mathrm{~ns}$. The energy calibration was performed using ${ }^{133} \mathrm{Ba}$ and ${ }^{137} \mathrm{C}$ s sources. The obtained typical energy resolution was $\sigma / E=4.4 \%$ at $662 \mathrm{keV}$.

The PSD analysis for the selection of neutron events in the EJ-309 detectors was based on the head/tail analysis and on the analysis of the mean time of the pulse. This latter is defined as: $\tau=\sum a_{i} \cdot t_{i} / \sum a_{i}$, where $a_{i}$ is the pulse height amplitude at the time $t_{i}$ and the sum is extended over the first $100 \mathrm{~ns}$ of the pulse. The used software energy threshold for $\mathrm{ZnWO}_{4}$ detector was $30 \mathrm{keVee}$. At this value, only the recoils of oxygen nuclei can be observed. In particular, considering the estimation of the quenching factors of Ref. [60], in these measurements, the $\mathrm{Zn}$ and $\mathrm{W}$ recoils are expected at energies between $7 \mathrm{keVee}$ and $14 \mathrm{keVee}$ and between $1.6 \mathrm{keVee}$ and $2.5 \mathrm{keVee}$, respectively. The searched event pattern was given by an event in the $\mathrm{ZnWO}_{4}$ detector in coincidence with a neutron in the EJ-309 detectors. A clear peak was observed in the $\mathrm{ZnWO}_{4}$ energy distribution, when a proper time of flight interval was selected for neutrons travelling from the $\mathrm{ZnWO}_{4}$ to the neutron detectors [12]. The quenching factors were evaluated from the positions of these peaks 
in the energy scale calibrated with $\gamma$ quanta. The measurements were repeated studying the nuclear recoils along the three $\mathrm{ZnWO}_{4}$ crystal axes and with different recoil energies (scattering angle). Since the responses of the crystal axes I and II are expected to be similar, only the case of axis I was considered. The measured quenching factors are shown in Figure 8 along with the models for the considered crystal axes obtained by fitting the $\alpha^{\prime} \mathrm{s}$ and oxygen recoil $(\mathrm{O})$ data with the prescription of Ref. [60] (see Ref. [12]).

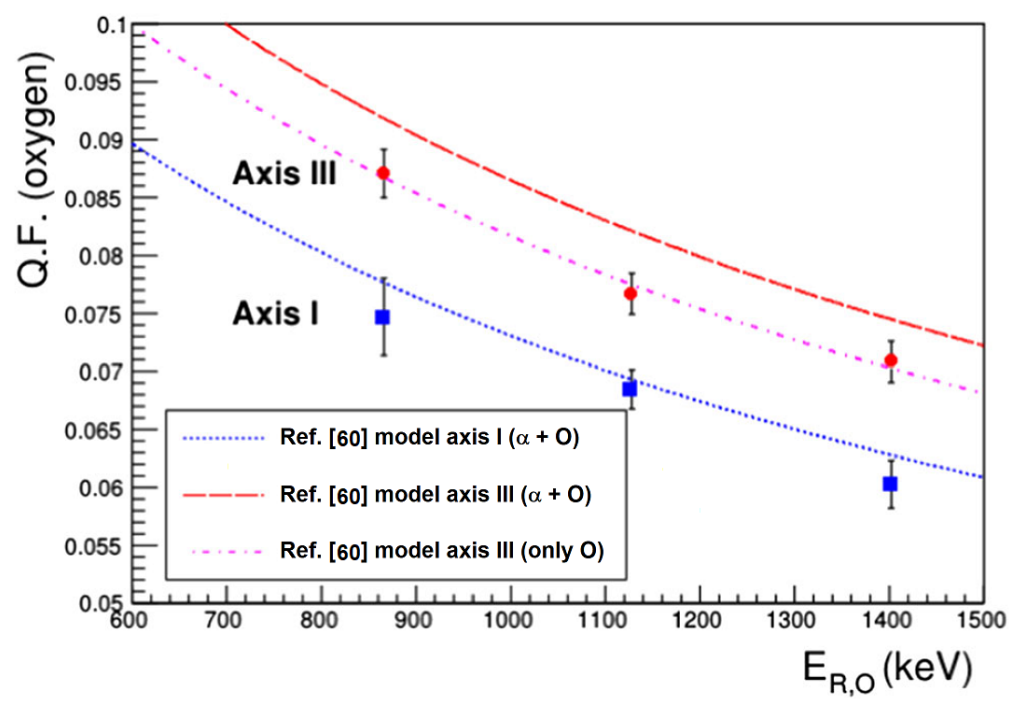

Figure 8. Quenching factors measured with a $\mathrm{ZnWO}_{4}$ detector in Ref. [12] for oxygen nuclear recoils along the crystal axes I and III as a function of the expected recoil energies $E_{R, O}$. The models for the considered crystal axes obtained by fitting the $\alpha$ 's and oxygen recoil $(\mathrm{O})$ data following the prescription of Ref. [60] are also reported. Reprinted with permission from [12]. Copyright 2020 Springer Nature.

A "mean anisotropy" equal to $(1.145 \pm 0.027)$ can be calculated considering the ratio between the quenching factors measured along axes III and I, averaged over the different recoil energies [12]. Thus, the anisotropy in the light response for oxygen nuclear recoils is significantly evident at the $5.4 \sigma$ confidence level. Moreover, the trend of the measured quenching factors supports the presence of an anisotropy at a level of $\sim 15 \%$ at $20 \mathrm{keV}$ recoil energy.

In conclusion, the results briefly reported here demonstrate the goodness of anisotropic features of the $\mathrm{ZnWO}_{4}$ scintillator. Moreover, the high radiopurity level reachable by this detector and its good potential characteristics in terms of light output when operated at a temperature of a few tens of degrees [58] makes it very competitive in the search for rare processes even at low energy. These results open the possibility to realize the pioneer ADAMO experiment and to exploit the directionality technique to investigate those DM candidate particles that induce recoils of target nuclei.

The R\&D for further radiopurification of $\mathrm{ZnWO}_{4}$ crystal scintillators and optimization of the light response is ongoing. In particular, the synthesis of the $\mathrm{ZnWO}_{4}$ compound from zinc oxides and tungsten oxides of different origin, including commercially available, was carried out and optimized. Optimal synthesis time and temperature were found and the analysis of the synthesized $\mathrm{ZnWO}_{4}$ compound for each process was done. Many growth processes have been carried out and crystals of single and double crystallizations were grown from the synthesized $\mathrm{ZnWO}_{4}$ charge from stoichiometric and un-stoichiometric melts. Optimization of $\mathrm{ZnWO}_{4}$ crystals annealing in an oxygen-containing atmosphere was done and the evaluation of crystal's optical quality dependence on initial $\mathrm{Zn}$ and $\mathrm{W}$ oxides purity levels, origins, synthesis procedures and stoichiometry was carried out. Further work will be aimed at obtaining larger crystals of high uniformity, optical quality, and free of various inclusions and defects. 


\section{5. $\mathrm{SrI}_{2}(\mathrm{Eu})$ Crystal Scintillator}

The strontium iodide, $\mathrm{SrI}_{2}(\mathrm{Eu})$, is another inorganic scintillator that has a high amount of potential in the field of DM and rare processes investigation. The strontium iodide scintillator was patented in 1968 by Hofstadter [62]. In the last few years, the high light output $(>100,000$ photons $/ \mathrm{MeV})$, the good energy resolution $(\approx 3 \%$ at $662 \mathrm{keV})$ and the possibility to grow crystals with large-size and uniform light yield have increased the interest in this detector [63-66]. The main properties of $\mathrm{SrI}_{2}(\mathrm{Eu})$ crystal scintillators are summarized in Table 1.

Table 1. Properties of $\mathrm{SrI}_{2}(\mathrm{Eu})$ crystal scintillators.

\begin{tabular}{lll}
\hline Property & Value & Reference \\
\hline Density $\left(\mathrm{g} / \mathrm{cm}^{3}\right)$ & $4.5-4.6$ & {$[63,65,67]$} \\
Melting point $\left({ }^{\circ} \mathrm{C}\right)$ & 515 & {$[63]$} \\
Structural type & Orthorhombic & {$[63]$} \\
Index of refraction & 1.85 & {$[68]$} \\
Wavelength of emission maximum $(\mathrm{nm})$ & $429-436$ & {$[63,65,67]$} \\
Light yield (photons $/ \mathrm{MeV})$ & $(68-120) \times 10^{3}$ & {$[63,65,69]$} \\
FWHM $(\%)$ for $662 \mathrm{keV} \gamma$ ray & $2.6-3.7$ & {$[63,65,69-71]$} \\
Scintillation decay time for $\gamma$ ray at $300 \mathrm{~K}(\mu \mathrm{s})$ & $0.6-2.4$ & {$[63,65,67,69]$} \\
\hline
\end{tabular}

An important point is that the matrix elements of $\operatorname{SrI}_{2}(\mathrm{Eu})$ crystal do not have natural long lived radioactive isotopes (an exception is ${ }^{84} \mathrm{Sr}$, isotopic abundance $0.56(2) \%$, which is a double $\beta$ decay candidate isotope, but this decay has never been observed and the half-life limits are of the order of $10^{16}$ y [13]). It is not the same for other crystal scintillators with high resolution like $\mathrm{LaCl}_{3}(\mathrm{Ce}), \mathrm{LaBr}_{3}(\mathrm{Ce}), \mathrm{LuI}_{3}(\mathrm{Ce})$, or $\mathrm{Lu}_{2} \mathrm{SiO}_{5}(\mathrm{Ce})$, which have long lived radioactive isotopes as ${ }^{138} \mathrm{La}$ and ${ }^{176} \mathrm{Lu}$. Moreover, thanks to the high light output, the $\mathrm{SrI}_{2}(\mathrm{Eu})$ scintillator has the potential to reach a very low energy threshold. These features make the $\mathrm{SrI}_{2}(\mathrm{Eu})$ detector promising in various applications, as the low counting experiments for the search of DM and double $\beta$ decay (the ${ }^{84} \mathrm{Sr}$, a double $\beta$ candidate isotope, is indeed contained in the detector itself).

The internal radioactive contamination of a $\operatorname{SrI}_{2}(\mathrm{Eu})$ crystal scintillator were studied in Ref. [13]. The used crystal was grown with the vertical Stockbarger method in a quartz ampoule [72]. In particular, the reaction of strontium carbonate and europium oxide with the hydroiodic acid [62] was used to obtain anhydrous strontium iodide activated by europium at $1.2 \%$. The obtained hydrate was dried, placed in a quartz ampoule for the crystal growth and slowly heated with permanent vacuum pumping. When a temperature of $150{ }^{\circ} \mathrm{C}$ was reached (after five days), the ampoule was welded and placed in the Stockbarger growing set-up. Then, the temperature was increased up to $538^{\circ} \mathrm{C}$ and the crystal was grown with a speed of $20 \mathrm{~mm}$ per day [65]. A near to cylindrical crystal $\oslash 13 \times 11 \mathrm{~mm}$ was cut from the crystal boule using a dry box filled by pure nitrogen. The crystal was neither milky nor cracked. After wrapping the crystal with PTFE tape, it was encapsulated in an oxygen-free high thermal conductivity (OFHC) copper housing using epoxy glue. A quartz window was present on one side of the housing to allow the collection of the scintillation light. All the materials used in the crystal assembling had a low level of radioactive contamination.

A low background set-up located at sea-level in the Institute for Nuclear Research of NASU (Ukraine) was used to measure the internal radioactive contamination of the $\mathrm{SrI}_{2}(\mathrm{Eu})$ crystal scintillator. A sketch of the set-up is shown in Figure 9.

The detector was optically coupled to a $3^{\prime \prime}$ PMT tube Philips XP2412 through a high purity polystyrene light-guide $\left(\oslash 66 \times 120 \mathrm{~mm}^{2}\right)$. A passive shield made of OFHC copper (5-12 cm thick) and lead ( $5 \mathrm{~cm}$ thick) surrounded the detector. Moreover, an anti-muon veto counter $(50 \times 50 \times 8 \mathrm{~cm}$ polystyrene based plastic scintillator) was installed above the 
set-up. The energy spectrum measured with the $\mathrm{SrI}_{2}(\mathrm{Eu})$ scintillator over $101.52 \mathrm{~h}$ together with a model of the background is presented in Figure 10-Left.

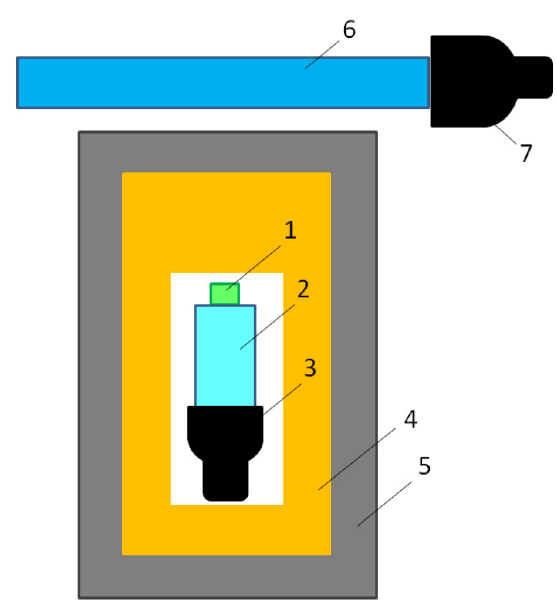

Figure 9. Schematic view of the experimental set-up with the $\mathrm{SrI}_{2}(\mathrm{Eu})$ scintillation detector. The $\mathrm{SrI}_{2}(\mathrm{Eu})$ crystal scintillator (1) is viewed through a polystyrene light-guide (2) by a photo-multiplier tube (3). The detector is surrounded by copper (4) and lead (5). The polystyrene based plastic scintillator of the anti-muon veto counter (6) is viewed by a photo-multiplier tube (7).
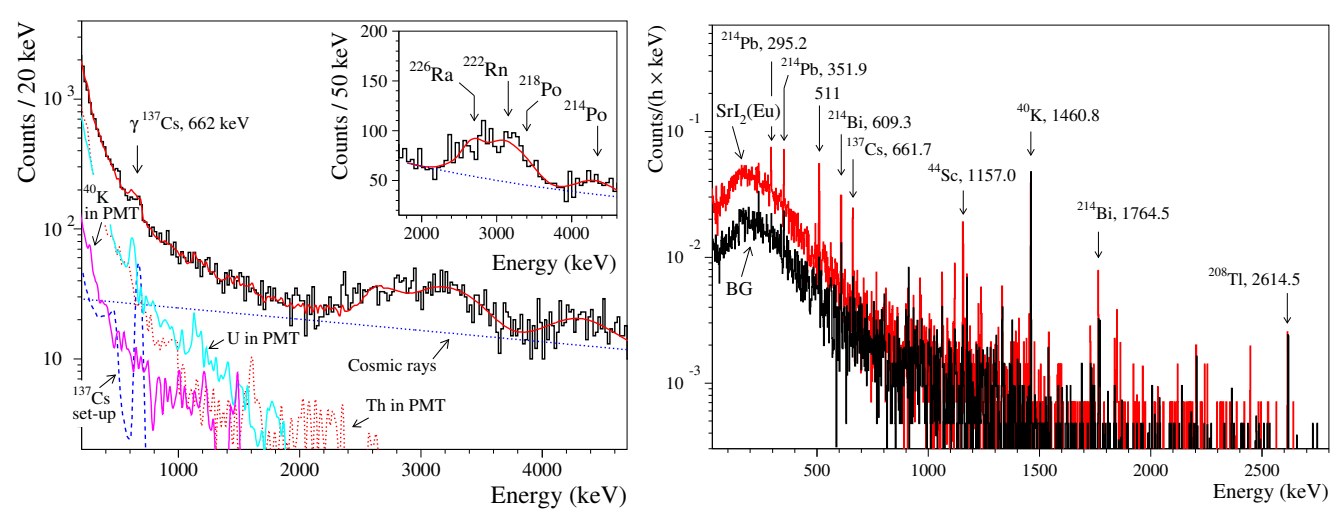

Figure 10. (Color online) Left: Energy spectrum measured with a $\operatorname{SrI}_{2}(\mathrm{Eu})$ crystal scintillator in Ref. [13] over $101.52 \mathrm{~h}$. The model of the background is also reported together with the main components of the external background and of the cosmic rays. (Inset) The structure in the region (2.5-4.3) MeV is due to $\alpha$ activity of trace U/Th contamination of the crystal scintillator. Right: Energy spectra measured by a ULB HPGe $\gamma$ spectrometer deep underground in Ref. [13]. The measurements were performed with the $\mathrm{SrI}_{2}(\mathrm{Eu})$ sample $(706 \mathrm{~h})$ and without it $(\mathrm{BG}, 1046 \mathrm{~h})$. The reported $\gamma$ lines energies are in $\mathrm{keV}$. The BG spectrum was measured before the contamination of the set-up by a ${ }^{44} \mathrm{Ti}$ $\left(1157 \mathrm{keV}\right.$ peak of $\left.{ }^{44} \mathrm{Sc}\right)$. Reprinted with permission from [73]. Copyright 2020 IOP Publishing.

The derived summary on activities is presented in Table 2 .

The data analysis also allowed the estimation of the $\alpha / \gamma$ ratio as 0.55 for the $\alpha$ particles of ${ }^{214} \mathrm{Po}\left(\mathrm{E}_{\alpha}=7687 \mathrm{keV}\right)$; moreover, no clear indication on differences was observed in the kinetics of the scintillation decay in the $\operatorname{SrI}_{2}(\mathrm{Eu})$ crystal scintillator under $\gamma$ quanta ( $\beta$ particles) and $\alpha$ particles irradiation.

The $\mathrm{SrI}_{2}(\mathrm{Eu})$ crystal was then measured with the ULB HPGe $\gamma$ ray spectrometer GeCris $\left(468 \mathrm{~cm}^{3}\right)$ located deep underground at the LNGS. This detector has a rather thin $\mathrm{Cu}$ window of $1 \mathrm{~mm}$ thickness. The passive shield of the detector consists of $15 \mathrm{~cm}$ of OFHC copper and $20 \mathrm{~cm}$ of low radioactive lead. The whole set-up is sealed in an air-tight plexiglass box continuously flushed with high purity nitrogen gas to avoid the presence of residual environmental radon. The data accumulated with and without the $\operatorname{SrI}_{2}(\mathrm{Eu})$ sample are shown in Figure 10-Right. The measured activities are reported in Table 2. 
Table 2. Radioactive contamination of the $\mathrm{SrI}_{2}(\mathrm{Eu})$ scintillator used in Ref. [13]. The upper limits are given at $90 \%$ C.L., and the uncertainties of the activities at $68 \%$ C.L.

\begin{tabular}{|c|c|c|c|}
\hline \multirow[b]{2}{*}{ Chain } & \multirow{2}{*}{$\begin{array}{l}\text { Nuclide } \\
\text { (Sub-Chain) }\end{array}$} & \multicolumn{2}{|c|}{ Activity (mBq/kg) } \\
\hline & & $\begin{array}{l}\text { Measured in } \\
\text { Scintillation Mode }\end{array}$ & Measured by HPGe \\
\hline \multirow{11}{*}{${ }^{232} \mathrm{Th}$} & ${ }^{40} \mathrm{~K}$ & $\leq 200$ & $\leq 255$ \\
\hline & ${ }^{60} \mathrm{Co}$ & $<540$ & $<16$ \\
\hline & ${ }^{90} \mathrm{Sr}-{ }^{90} \mathrm{Y}$ & $<90$ & \\
\hline & ${ }^{137} \mathrm{Cs}$ & $\leq 140$ & $53 \pm 11$ \\
\hline & ${ }^{138} \mathrm{La}$ & $<1100$ & $<20$ \\
\hline & ${ }^{152} \mathrm{Eu}$ & $\leq 840$ & $\leq 108$ \\
\hline & ${ }^{154} \mathrm{Eu}$ & $<910$ & $<67$ \\
\hline & ${ }^{176} \mathrm{Lu}$ & $\leq 970$ & $\leq 143$ \\
\hline & ${ }^{232} \mathrm{Th}$ & $\leq 3$ & \\
\hline & ${ }^{228} \mathrm{Ac}$ & & $\leq 68$ \\
\hline & ${ }^{228} \mathrm{Th}$ & $6 \pm 2$ & $\leq 52$ \\
\hline \multirow[t]{4}{*}{${ }^{238} \mathrm{U}$} & ${ }^{238} \mathrm{U}$ & $<40$ & \\
\hline & ${ }^{226} \mathrm{Ra}$ & $100 \pm 14$ & $120 \pm 50$ \\
\hline & ${ }^{210} \mathrm{~Pb}-{ }^{210} \mathrm{Bi}$ & $\leq 180$ & \\
\hline & ${ }^{210} \mathrm{Po}$ & $\leq 60$ & \\
\hline
\end{tabular}

The intrinsic radiopurity of the $\mathrm{SrI}_{2}(\mathrm{Eu})$ scintillator is still far from that of the $\mathrm{NaI}(\mathrm{Tl})$ scintillators [18]. On the other hand, it is three orders of magnitude better than that of other high light output scintillators as those containing $\mathrm{La}$, as $\mathrm{LaCl}_{3}(\mathrm{Ce})$ and $\mathrm{LaBr}_{3}(\mathrm{Ce})$, and five orders of magnitude better than that of the scintillators containing $\mathrm{Lu}$, as $\mathrm{Lu}_{2} \mathrm{SiO}_{5}(\mathrm{Ce})$ and $\mathrm{LuI}_{3}(\mathrm{Ce})$.

A better radiopurity for the $\mathrm{SrI}_{2}(\mathrm{Eu})$ crystal can be reached by selecting the starting $\mathrm{SrI}_{2}$ and $\mathrm{EuI}_{2}$ materials and applying efficient purification procedures. Moreover, the performances and the ability to grow high quality, uncracked $\mathrm{SrI}_{2}(\mathrm{Eu})$ crystals also strongly depend on the quality of the starting components. Often, the zone refining is used to enhance the material purity, particularly for the $\mathrm{EuI}_{2}$ dopant, which cannot be obtained commercially in an ultra-high purity form $[74,75]$.

Another aspect that can affect the light yield and light collection uniformity [15] of $\mathrm{SrI}_{2}(\mathrm{Eu})$ crystal is the Eu doping concentration [76]. The scintillation properties of pure $\mathrm{SrI}_{2}$ and $\mathrm{SrI}_{2}$ doped with different europium concentration were studied e.g., in Refs. [67,69]. In $\mathrm{Eu}^{2+}$ doped crystals, light-trapping can occur, in which scintillation light is re-absorbed and re-emitted. It complicates the acquisition of high resolution gamma ray spectra but is readily mitigated with a small taper applied to the crystal and/or digital signal processing [66].

As shown in Ref. [77], it is possible to further improve the light output and the energy resolution also lowering the working temperature. In particular, it has been shown that the already excellent scintillation properties are particularly enhanced in the lower temperature range of 130 to $180 \mathrm{~K}$, with a reduction in decay time and increase in light output by approximately $50 \%$ and $35 \%$, respectively. In Figure 11, the light output, normalized to its value at $295 \mathrm{~K}$, is shown as a function of temperature. The relative light output increased as the temperature was decreased from room temperature towards $130 \mathrm{~K}$, with the maximum occurring between 130 and $180 \mathrm{~K}$, where the emission intensity was also observed to be the greatest. The increasing light output may be attributed to the reduction in non-radiative processes which typically dominate and reduce luminescence in alkali halides at higher (i.e., ambient) temperatures [77].

In conclusion, the results of these studies demonstrate the applicability of $\operatorname{SrI}_{2}(\mathrm{Eu})$ detectors to a variety of applications, and show the potential perspective of this highly efficient scintillator for low counting measurements, such as those for the investigation of DM and double $\beta$ decay of ${ }^{84} \mathrm{Sr}$. 


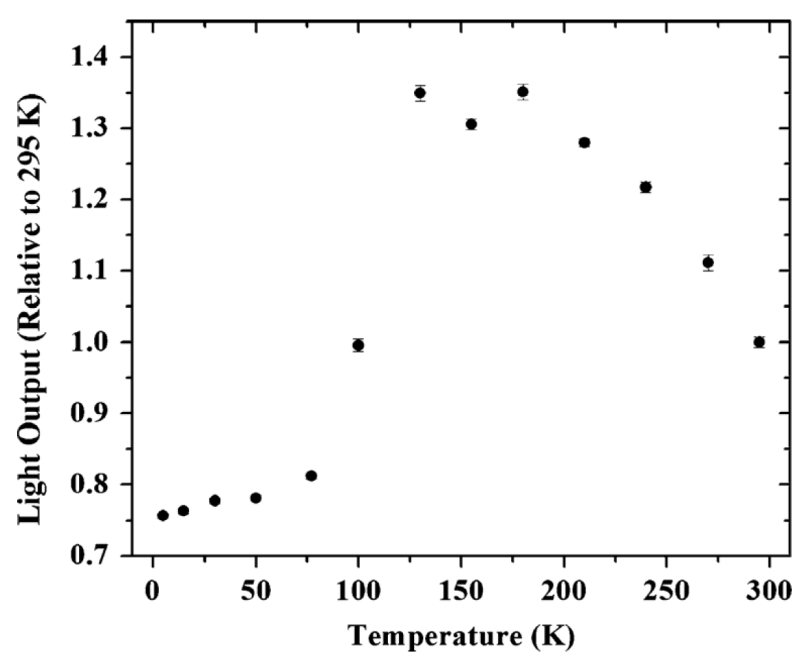

Figure 11. Evolution of estimated light output as a function of temperature for $\operatorname{SrI}_{2}(\mathrm{Eu})$ crystal scintillator under ${ }^{137} \mathrm{Cs}$ gamma-ray excitation, as measured in Ref. [77]. Reprinted with permission from [77]. Copyright 2012 IEEE.

\section{Conclusions}

In this paper, some of the important roles and the perspectives of inorganic scintillators in the investigation of DM have been discussed. The many advantages offered by these detectors and the possibility to develop ULB crystals make them particularly suited for the study of model independent DM signatures, as the DM-AM, and for the directionality technique, in the case of detectors with anisotropic response.

In the field of direct DM investigation, three of the most interesting inorganic crystal scintillators are $\mathrm{NaI}(\mathrm{Tl}), \mathrm{ZnWO}_{4}$ and $\mathrm{SrI}_{2}(\mathrm{Eu})$. Their properties, potential and the recent results obtained by DAMA for $\mathrm{NaI}(\mathrm{Tl})$ detectors and by the collaboration between DAMA and the Institute for Nuclear Research of NASU (Ukraine), for $\mathrm{ZnWO}_{4}$ and $\mathrm{SrI}_{2}(\mathrm{Eu})$ detectors, have been reviewed.

Author Contributions: The authors have been significantly contributing to the conceptualization, and writing of the paper. All authors have read and agreed to the published version of the manuscript.

Funding: Fedor Danevich, Dmytro Kasperovych, and Vladimir Tretyak were supported in part by the National Research Foundation of Ukraine Grant No. 2020.02/0011.

Acknowledgments: The authors are grateful to all the members of DAMA collaboration and to the researchers from the Institute for Nuclear Research of NASU (Kyiv, Ukraine) and other institutions who have contributed to the investigations reviewed in this paper.

Conflicts of Interest: The authors declare no conflict of interest.

\section{References}

1. Drukier, A.K.; Freese, K.; Spergel, D.N. Detecting cold dark-matter candidates. Phys. Rev. D 1986, 33, 3495. [CrossRef]

2. Freese, K.; Frieman, J.; Gould, A. Signal modulation in cold-dark-matter detection. Phys. Rev. D 1988, 37, 3388. [CrossRef]

3. Bernabei, R.; Belli, P.; Cappella, F.; Cerulli, R.; Montecchia, F.; Nozzoli, F.; Incicchitti, A.; Prosperi, D.; Dai, C.J.; Kuang, H.H.; et al. Dark matter search. La Rivista del Nuovo Cimento 2003, 26, 1-73. [CrossRef]

4. Bernabei, R.; Belli, P.; Cappella, F.; Cerulli, R.; Dai, C.J.; d'Angelo, A.; He, H.L.; Incicchitti, A.; Kuang, H.H.; Ma, J.M.; et al. First results from DAMA/LIBRA and the combined results with DAMA/NaI. Eur. Phys. J. C 2008, 56, 333-355. [CrossRef]

5. Bernabei, R.; Belli, P.; Cappella, F.; Cerulli, R.; Dai, C.J.; d'Angelo, A.; He, H.L.; Incicchitti, A.; Kuang, H.H.; Ma, X.H.; et al. New results from DAMA/LIBRA. Eur. Phys. J. C 2010, 67, 39-49. [CrossRef]

6. Bernabei, R.; Belli, P.; Cappella, F.; Caracciolo, V.; Castellano, S.; Cerulli, R.; Dai, C.J.; d'Angelo, A.; d'Angelo, S.; Di Marco, A.; et al. Final model independent result of DAMA/LIBRA-phase1. Eur. Phys. J. C 2013, 73, 2648. [CrossRef]

7. Bernabei, R.; Belli, P.; Bussolotti, A.; Cappella, F.; Caracciolo, V.; Cerulli, R.; Dai, C.J.; d'Angelo, A.; Di Marco, A.; He, H.L.; et al. First model independent results from DAMA/LIBRA-phase2. Nucl. Phys. At. Energy 2018, 19, 307-325. [CrossRef] 
8. Bernabei, R.; Belli, P.; Cappella, F.; Caracciolo, V.; Cerulli, R.; Dai, C.J.; d'Angelo, A.; Di Marco, A.; He, H.L.; Incicchitti, A.; et al. Improved model-dependent corollary analyses after the first six annual cycles of DAMA/LIBRA-phase2. Nucl. Phys. At. Energy 2019, 20, 317-348. [CrossRef]

9. Spergel, D.N. Motion of the Earth and the detection of weakly interacting massive particles. Phys. Rev. D 1988, 37, 1353. [CrossRef]

10. Cappella, F.; Bernabei, R.; Belli, P.; Caracciolo, V.; Cerulli, R.; Danevich, F.A.; d'Angelo, A.; Di Marco, A.; Incicchitti, A.; Poda, D.V.; et al. On the potentiality of the $\mathrm{ZnWO}_{4}$ anisotropic detectors to measure the directionality of Dark Matter. Eur. Phys. J. C 2013, 73, 2276. [CrossRef]

11. Belli, P.; Bernabei, R.; Borovlev, Y.A.; Cappella, F.; Caracciolo, V.; Cerulli, R.; Danevich, F.A.; Incicchitti, A.; Kasperovych, D.V.; Polischuk, O.G.; et al. New development of radiopure $\mathrm{ZnWO}_{4}$ crystal scintillators. Nucl. Instrum. Meth. A 2019, 935, 89-94. [CrossRef]

12. Belli, P.; Bernabei, R.; Cappella, F.; Caracciolo, V.; Cerulli, R.; Cherubini, N.; Danevich, F.A.; Incicchitti, A.; Kasperovych, D.V.; Merlo, V.; et al. Measurements of $\mathrm{ZnWO}_{4}$ anisotropic response to nuclear recoils for the ADAMO project. Eur. Phys. J. A 2020, 56, 83. [CrossRef]

13. Belli, P.; Bernabei, R.; Cerulli, R.; Danevich, F.A.; Galenin, E.; Gektin, A.; Incicchitti, A.; Isaienko, V.; Kobychev, V.V.; Laubenstein, M.; et al. Radioactive contamination of $\mathrm{SrI}_{2}(\mathrm{Eu})$ crystal scintillator. Nucl. Instrum. Meth. A 2012, 670, 10-17. [CrossRef]

14. Hirsch, M.; Muto, K.; Oda, T.; Klapdor-Kleingrothaus, H.V. Nuclear structure calculation of $\beta^{+} \beta^{+}, \beta^{+} / \mathrm{EC}$ and EC/EC decay matrix elements. Z. Phys. A 1994, 347, 151-160.

15. Danevich, F.A.; Tretyak, V.I. Radioactive contamination of scintillators. Int. J. Mod. Phys. A 2018, 33, 1843007. [CrossRef]

16. Bernabei, R.; Belli, P.; Incicchitti, A.; Dai, C.J. Adopted low background techniques and analysis of radioactive trace impurities. Int. J. Mod. Phys. A 2016, 31, 1642003. [CrossRef]

17. Barabash, A.S.; Belli, P.; Bernabei, R.; Borovlev, Y.A.; Cappella, F.; Caracciolo, V.; Cerulli, R.; Danevich, F.A.; Incicchitti, A.; Kobychev, V.V.; et al. Improvement of radiopurity level of enriched ${ }^{116} \mathrm{CdWO}_{4}$ and $\mathrm{ZnWO}_{4}$ crystal scintillators by recrystallization. Nucl. Instrum. Meth. A 2016, 833, 77-81. [CrossRef]

18. Bernabei, R.; Belli, P.; Bussolotti, A.; Cappella, F.; Cerulli, R.; Dai, C.J.; d'Angelo, A.; He, H.L.; Incicchitti, A.; Kuang, H.H.; et al. The DAMA/LIBRA apparatus. Nucl. Instr. Meth. A 2008, 592, 297-315. [CrossRef]

19. Bernabei, R.; Belli, P.; Landoni, V.; Montecchia, F.; Di Nicolantonio, W.; Incicchitti, A.; Prosperi, D.; Bacci, C.; Dai, C.J.; Ding, L.K.; et al. New limits on WIMP search with large-mass low-radioactivity NaI(Tl) set-up at Gran Sasso. Phys. Lett. B 1996, 389, 757-766. [CrossRef]

20. See the Publication List. Available online: http:// people.roma2.infn.it/dama (accessed on 27 April 2021).

21. Bernabei, R.; Belli, P.; Bussolotti, A.; Cappella, F.; Caracciolo, V.; Casalboni, M.; Cerulli, R.; Dai, C.J.; d'Angelo, A.; Di Marco, A.; et al. Performances of the new high quantum efficiency PMTs in DAMA/LIBRA. JINST 2012, 7, P03009. [CrossRef]

22. Bernabei, R.; Incicchitti, A. Low background techniques in NaI(Tl) setups. Int. J. Mod. Phys. A 2017, 32, 1743007. [CrossRef]

23. Bernabei, R.; Belli, P.; Bussolotti, A.; Cerulli, R.; Di Marco, A.; Merlo, V.; Montecchia, F.; Cappella, F.; d'Angelo, A.; Incicchitti, A.; et al. New model independent results from the first six full annual cycles of DAMA/LIBRA-phase2. Bled Work. Phys. 2018, 19, 27-57.

24. Bernabei, R.; Belli, P.; d'Angelo, S.; Di Marco, A.; Montecchia, F.; Cappella, F.; d'Angelo, A.; Incicchitti, A.; Caracciolo, V.; Castellano, S.; et al. Dark matter investigation by DAMA at Gran Sasso. Int. J. Mod. Phys. A 2013, 28, 1330022. [CrossRef]

25. Smith, D.; Weiner, N. Inelastic dark matter. Phys. Rev. D 2001, 64, 043502. [CrossRef]

26. Tucker-Smith, D.; Weiner, N. Status of inelastic dark matter. Phys. Rev. D 2005, 72, 063509. [CrossRef]

27. Finkbeiner, D.P.; Lin, T.; Weiner, N. Inelastic dark matter and DAMA/LIBRA: An experimentum crucis. Phys. Rev. D 2009, 80, 115008. [CrossRef]

28. Freese, K.; Gondolo, P.; Newberg, H.J. Detectability of weakly interacting massive particles in the Sagittarius dwarf tidal stream. Phys. Rev. D 2005, 71, 043516. [CrossRef]

29. Freese, K.; Gondolo, P.; Newberg, H.J.; Lewis, M. Effects of the Sagittarius dwarf tidal stream on dark matter detectors. Phys. Rev. Lett. 2004, 92, 111301. [CrossRef] [PubMed]

30. Bernabei, R.; Belli, P.; Bussolotti, A.; Cappella, F.; Caracciolo, V.; Cerulli, R.; Dai, C.J.; d'Angelo, A.; Di Marco, A.; Ferrari, N.; et al. The DAMA project: Achievements, implications and perspectives. Prog. Part. Nucl. Phys. 2020, 114, 103810. [CrossRef]

31. Bernabei, R.; Belli, P.; Bussolotti, A.; Cappella, F.; Caracciolo, V.; Cerulli, R.; Dai, C.J.; d'Angelo, A.; Di Marco, A.; He, H.L.; et al. First model independent results from DAMA/LIBRA-phase2. Universe 2018, 4, 116. [CrossRef]

32. Bernabei, R.; Belli, P.; Cappella, F.; Cerulli, R.; Montecchia, F.; Nozzoli, F.; Incicchitti, A.; Prosperi, D.; Dai, C.J.; Kuang, H.H.; et al. Dark matter particles in the galactic halo: Results and implications from DAMA/NaI. Int. J. Mod. Phys. D 2004, 13, $2127-2159$. [CrossRef]

33. Bernabei, R.; Belli, P.; d'Angelo, S.; Di Marco, A.; Montecchia, F.; d'Angelo, A.; Incicchitti, A.; Prosperi, D.; Cappella, F.; Caracciolo, V.; et al. DAMA/LIBRA-phase1 model independent results. Int. J. Mod. Phys. A 2016, 31, 1642006. [CrossRef]

34. Bernabei, R.; Belli, P.; Cappella, F.; Caracciolo, V.; Cerulli, R.; Dai, C.J.; d'Angelo, A.; d'Angelo, S.; Di Marco, A.; He, H.L.; et al. No role for neutrons, muons and solar neutrinos in the DAMA annual modulation results. Eur. Phys. J. C 2014, 74, 3196. [CrossRef]

35. Bernabei, R.; Belli, P.; Cerulli, R.; Montecchia, F.; Amato, M.; Ignesti, G.; Incicchitti, A.; Prosperi, D.; Dai, C.J.; He, H.L.; et al. On the investigation of possible systematics in WIMP annual modulation search. Eur. Phys. J. C 2000, 18, 283-292. [CrossRef] 
36. Bernabei, R.; Belli, P.; Cappella, F.; Caracciolo, V.; Cerulli, R.; Dai, C.J.; d'Angelo, A.; Di Marco, A.; He, H.L.; Incicchitti, A.; et al. No role for muons in the DAMA annual modulation results. Eur. Phys. J. C 2012, 72, 2064. [CrossRef]

37. Davis, J.H. Fitting the Annual Modulation in DAMA with Neutrons from Muons and Neutrinos. Phys. Rev. Lett. 2014, 113, 081302. [CrossRef]

38. Bernabei, R.; Belli, P.; Montecchia, F.; De Sanctis, M.; Di Nicolantonio, W.; Incicchitti, A.; Prosperi, D.; Bacci, C.; Dai, C.J.; Ding, L.K.; et al. Search for non-paulian transitions in ${ }^{23} \mathrm{Na}$ and ${ }^{127} \mathrm{I}$. Phys. Lett. B 1997, 408, 439-444. [CrossRef]

39. Belli, P.; Bernabei, R.; Dai, C.J.; He, H.L.; Ignesti, G.; Incicchitti, A.; Kuang, H.H.; Ma, J.M.; Montecchia, F.; Ponkratenko, O.A.; et al. New experimental limit on the electron stability and non-paulian transitions in Iodine atoms. Phys. Lett. B 1999, 460, $236-241$. [CrossRef]

40. Bernabei, R.; Belli, P.; Cerulli, R.; Montecchia, F.; Amato, M.; Ignesti, G.; Incicchitti, A.; Prosperi, D.; Dai, C.J.; He, H.L.; et al. Extended limits on neutral strongly interacting massive particles and nuclearites from NaI(Tl) scintillators. Phys. Rev. Lett. 1999, 83, 4918. [CrossRef]

41. Belli, P.; Bernabei, R.; Dai, C.J.; He, H.L.; Ignesti, G.; Incicchitti, A.; Kuang, H.H.; Ma, J.M.; Montecchia, F.; Ponkratenko, O.A.; et al. New limits on the nuclear levels excitation of ${ }^{127} \mathrm{I}$ and ${ }^{23} \mathrm{Na}$ during charge nonconservation. Phys. Rev. C 1999, 60, 065501. [CrossRef]

42. Bernabei, R.; Belli, P.; Cerulli, R.; Montecchia, F.; Nozzoli, F.; Incicchitti, A.; Prosperi, D.; Dai, C.J.; He, H.L.; Kuang, H.H.; et al. Search for solar axions by Primakoff effect in NaI crystals. Phys. Lett. B 2001, 515, 6-12. [CrossRef]

43. Cappella, F.; Cerulli, R.; Incicchitti, A. A preliminary search for Q-balls by delayed coincidences in NaI(Tl). Eur. Phys. J. Direct C 2002, 14, 1-6. [CrossRef]

44. Bernabei, R.; Belli, P.; Cappella, F.; Montecchia, F.; Nozzoli, F.; d’Angelo, A.; Incicchitti, A.; Prosperi, D.; Cerulli, R.; Dai, C.J.; et al. Search for spontaneous transition of nuclei to a superdense state. Eur. Phys. J. A 2005, 23, 7-10. [CrossRef]

45. Bernabei, R.; Belli, P.; Cappella, F.; Montecchia, F.; Nozzoli, F.; d'Angelo, A.; Incicchitti, A.; Prosperi, D.; Cerulli, R.; Dai, C.J.; et al. A search for spontaneous emission of heavy clusters in the ${ }^{127}$ I nuclide. Eur. Phys. J. A 2005, 24, 51-56. [CrossRef]

46. Bernabei, R.; Belli, P.; Cappella, F.; Cerulli, R.; Dai, C.J.; d'Angelo, A.; He, H.L.; Incicchitti, A.; Kuang, H.H.; Ma, X.H.; et al. New search for processes violating the Pauli exclusion principle in sodium and in iodine. Eur. Phys. J. C 2009, 62, 327-332. [CrossRef]

47. Bernabei, R.; Belli, P.; Cappella, F.; Cerulli, R.; Dai, C.J.; d'Angelo, A.; d'Angelo, S.; Di Marco, A.; He, H.L.; Incicchitti, A.; et al. Search for charge non-conserving processes in ${ }^{127} \mathrm{I}$ by coincidence technique. Eur. Phys. J. C 2012, 72, 1920. [CrossRef]

48. Bernabei, R.; Belli, P.; Cappella, F.; Caracciolo, V.; Castellano, S.; Cerulli, R.; Dai, C.J.; d'Angelo, A.; Di Marco, A.; He, H.L.; et al. New search for correlated $\mathrm{e}^{+} \mathrm{e}^{-}$pairs in the $\alpha$ decay of ${ }^{241}$ Am. Eur. Phys. J. A 2013, 49, 64. [CrossRef]

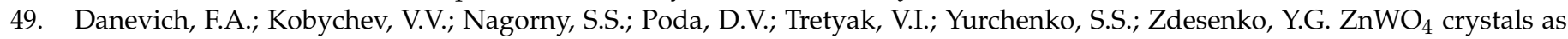
detectors for $2 \beta$ decay and dark matter experiments. Nucl. Instrum. Meth. A 2005, 544, 553-564. [CrossRef]

50. Belli, P.; Bernabei, R.; Bacci, C.; Incicchitti, A.; Prosperi, D. Identifying a "dark matter" signal by non-isotropic scintillation detector. Il Nuovo Cimento C 1992, 15, 473-479. [CrossRef]

51. Bernabei, R.; Belli, P.; Nozzoli, F.; Incicchitti, A. Anisotropic scintillators for WIMP direct detection: Revisited. Eur. Phys. J. C 2003, 28, 203-209. [CrossRef]

52. Belli, P.; Bernabei, R.; Cappella, F.; Cerulli, R.; Danevich, F.A.; Dubovik, A.M.; d'Angelo, S.; Galashov, E.N.; Grinyov, B.V.; Incicchitti, A.; et al. Radioactive contamination of $\mathrm{ZnWO}_{4}$ crystal scintillators. Nucl. Instrum. Meth. A 2011, 626-627, 31-38. [CrossRef]

53. Belli, P.; Bernabei, R.; Cappella, F.; Cerulli, R.; Danevich, F.A.; d'Angelo, S.; Incicchitti, A.; Kobychev, V.V.; Poda, D.V.; Tretyak, V.I. Final results of experiment to search for $2 \beta$ processes in zinc and tungsten with the help of radiopure $\mathrm{ZnWO}_{4}$ crystal scintillators. J. Phys. G Nucl. Part. Phys. 2011, 38, 115107. [CrossRef]

54. Galashov, E.N.; Gusev, V.A.; Shlegel, V.N.; Vasiliev, Y.V. Growing of $\mathrm{ZnWO}_{4}$ single crystals from melt by the low thermal gradient Czochralski technique. Funct. Mater. 2009, 16, 63-66.

55. Belli, P.; Bernabei, R.; Cappella, F.; Cerulli, R.; Danevich, F.A.; Grinyov, B.V.; Incicchitti, A.; Kobychev, V.V.; Mokina, V.M.; Nagorny, S.S.; et al. Search for double beta decay of zinc and tungsten with low background $\mathrm{ZnWO}_{4}$ crystal scintillators. Nucl. Phys. A 2009, 826, 256-273. [CrossRef]

56. Belli, P.; Bernabei, R.; Cappella, F.; Cerulli, R.; Dai, C.J.; Danevich, F.A.; Grinyov, B.V.; Incicchitti, A.; Kobychev, V.V.; Nagornaya, L.L.; et al. Search for $2 \beta$ processes in ${ }^{64} \mathrm{Zn}$ with the help of $\mathrm{ZnWO}_{4}$ crystal scintillator. Phys. Lett. B 2008, 658, 193-197. [CrossRef]

57. Belli, P.; Bernabei, R.; Cappella, F.; Cerulli, R.; Danevich, F.A.; Denisov, V.Y.; d'Angelo, A.; Incicchitti, A.; Kobychev, V.V.; Poda, D.V.; et al. Search for long-lived superheavy eka-tungsten with radiopure $\mathrm{ZnWO}_{4}$ crystal scintillator. Phys. Scr. 2015, $90,085301$. [CrossRef]

58. Nagornaya, L.L.; Danevich, F.A.; Dubovik, A.M.; Grinyov, B.V.; Henry, S.; Kapustyanyk, V.; Kraus, H.; Poda, D.V.; Kudovbenko, V.M.; Mikhailik, V.B.; et al. Tungstate and Molybdate Scintillators to Search for Dark Matter and Double Beta Decay. IEEE Trans. Nucl. Sci. 2009, 56, 2513-2518. [CrossRef]

59. Shlegel, V.N.; Borovlev, Y.A.; Grigoriev, D.N.; Grigorieva, V.D.; Danevich, F.A.; Ivannikova, N.V.; Postupaeva, A.G.; Vasiliev, Y.V. Recent progress in oxide scintillation crystals development by low-thermal gradient Czochralski technique for particle physics experiments. JINST 2017, 12, C08011. [CrossRef]

60. Tretyak, V.I. Semi-empirical calculation of quenching factors for ions in scintillators. Astropart. Phys. 2010, 33, 40-53. [CrossRef]

61. Birks, J.B. The Theory and Practice of Scintillation Counting; Pergamon Press: Oxford, UK, 1964. 
62. Hofstadter, R. Europium Activated Strontium Iodide Scintillators. U.S. Patent US3373279A, 12 March 1968.

63. Cherepy, N.J.; Hull, G.; Drobshoff, A.D.; Payne, S.A.; van Loef, E.; Wilson, C.M.; Shah, K.S.; Roy, U.N.; Burger, A.; Boatner, L.A.; et al. Strontium and barium iodide high light yield scintillators. Appl. Phys. Lett. 2008, 92, 083508. [CrossRef]

64. Cherepy, N.J.; Payne, S.A.; Asztalos, S.J.; Hull, G.; Kuntz, J.D.; Niedermayr, T.; Pimputkar, S.; Roberts, J.J.; Sanner, R.D.; Tillotson, T.M.; et al. Scintillators with potential to supersede lanthanum bromide. IEEE Trans. Nucl. Sci. 2009, 56, 873-880. [CrossRef]

65. van Loef, E.V.; Wilson, C.M.; Cherepy, N.J.; Hull, G.; Payne, S.A.; Choong, W.-S.; Moses, W.W.; Shah, K.S. Crystal growth and scintillation properties of strontium iodide scintillators. IEEE Trans. Nucl. Sci. 2009, 56, 869-872. [CrossRef]

66. Cherepy, N.J.; Beck, P.R.; Payne, S.A.; Swanberg, E.L.; Wihl, B.M.; Fisher, S.E.; Hunter, S.; Thelin, P.A.; Delzer, C.J.; Shahbazi, S.; et al. History and current status of strontium iodide scintillators. Proc. SPIE 2017, 10392, 1039202.

67. Alekhin, M.S.; de Haas, J.T.; Kramer, K.W.; Dorenbos, P. Scintillation properties of and self absorption in SrI 2 :Eu ${ }^{2+}$. IEEE Trans. Nucl. Sci. 2011, 58, 2519-2527. [CrossRef]

68. Tan, H.; Warburton, W.K. Modeling scintillation light absorption and re-emission in $\mathrm{SrI}_{2}(\mathrm{Eu})$ scintillators. Nucl. Instrum. Meth. A 2011, 652, 221-225. [CrossRef]

69. Glodo, J.; van Loef, E.V.; Cherepy, N.J.; Payne, S.A.; Shah, K.S. Concentration effects in Eu doped SrI 2. IEEE Trans. Nucl. Sci. 2010, 57, 1228-1232. [CrossRef]

70. Sturm, B.W.; Cherepy, N.J.; Drury, O.B.; Thelin, P.A.; Fisher, S.E.; Payne, S.A.; Burger, A.; Boatner, L.A.; Ramey, J.O.; Shah, K.S.; et al. Effects of packaging $\mathrm{SrI}_{2}(\mathrm{Eu})$ scintillator crystals. Nucl. Instr. Meth. A 2011, 652, 242-246. [CrossRef]

71. Cherepy, N.J.; Sturm, B.W.; Drury, O.B.; Hurst, T.A.; Sheets, S.A.; Ahle, L.E.; Saw, C.K.; Pearson, M.A.; Payne, S.A.; Burger, A.; et al. $\mathrm{SrI}_{2}$ scintillator for gamma ray spectroscopy. Proc. SPIE 2009, 7449, 74490F.

72. Stockbarger, D.C. The production of large single crystals of lithium fluoride. Rev. Sci. Instrum. 1936, 7, 133-136. [CrossRef]

73. Barabash, A.S.; Belli, P.; Bernabei, R.; Cappella, F.; Caracciolo, V.; Cerulli, R.; Danevich, F.A.; Di Marco, A.; Incicchitti, A.; Kasperovych, D.V.; et al. Low background scintillators to investigate rare processes. JINST 2020, 15, C07037. [CrossRef]

74. Calvert, G.; Guguschev, C.; Burger, A.; Groza, M.; Derby, J.J.; Feigelson, R.S. High speed growth of SrI 2 scintillator crystals by the EFG process. J. Cryst. Growth 2016, 455, 143-151. [CrossRef]

75. Arumugam, R.; Daniel, D.J.; Ramasamy, P. The purification process on scintillator material (SrI 2 :Eu) by zone-refinement technique. AIP Conf. Proc. 2015, 1665, 100019.

76. Raja, A.; Daniel, D.J.; Ramasamy, P.; Singh, S.G.; Sen, S.; Gadkari, S.C. Difficulties and improvement in growth of Europium doped Strontium Iodide $\left(\mathrm{SrI}_{2}: \mathrm{Eu}^{2+}\right)$ scintillator single crystal for radiation detection applications. J. Alloys Compd. 2018, 747, 989-993. [CrossRef]

77. Lam, S.; Gascon, M.; Hawrami, R.; Setyawan, W.; Curtarolo, S.; Feigelson, R.S.; Gaume, R.M. Nonproportionality and Scintillation Studies of Eu:SrI 2 from 295 to 5 K. IEEE Trans. Nucl. Sci. 2012, 59, 2052-2056. [CrossRef] 